\title{
Parity violation in electron scattering
}

\author{
P. Souder ${ }^{1, \dagger}$, K. D. Paschke ${ }^{2}$ \\ ${ }^{1}$ Syracuse University, Syracuse, NY 13244, USA \\ ${ }^{2}$ University of Virginia, Charlottesville, VA 22904, USA \\ Corresponding author.E-mail: †pasouder@syr.edu \\ Received August 31, 2015; accepted September 25, 2015
}

\begin{abstract}
By comparing the cross sections for left- and right-handed electrons scattered from various unpolarized nuclear targets, the small parity-violating asymmetry can be measured. These asymmetry data probe a wide variety of important topics, including searches for new fundamental interactions and important features of nuclear structure that cannot be studied with other probes. A special feature of these experiments is that the results are interpreted with remarkably few theoretical uncertainties, which justifies pushing the experiments to the highest possible precision. To measure the small asymmetries accurately, a number of novel experimental techniques have been developed.
\end{abstract}

Keywords weak neutral currents, weak form factors, parton distributions, neutron stars, physics beyond the standard model

PACS numbers 11.30.Er, 13.40.GP, 24.80.+y, 25.30.Bf

Contents

1 Introduction

1.1 History of the field

1.2 Experimental overview

1.3 Neutrino scattering and PVES

1.4 Atomic parity violation

1.5 Theory of parity violation in potential scattering

1.6 More realistic cases

1.7 Precision of SM predictions

1.7.1 Higher-order corrections

1.7.2 Uncertainties in radiative corrections

1.7.3 Experimental implications

2 Theory

2.1 Kinematics

2.2 Phenomenology

2.3 Weak charges

2.4 Strange form factors

2.5 Radius of neutron distribution

2.6 Parity violation in deep inelastic scattering

2.6.1 PVDIS for deuterium

2.6.2 Charge symmetry

2.6.3 Higher twist

2.6.4 PVDIS with a proton target

2.7 Møller scattering

*Special Topic: Spin Physics (Eds. Haiyan Gao \& Bo-Qiang Ma).

3 Experimental details

3.1 Rates and statistical errors

2

2

3

4

4

4

5

5

6

6

6

6

6

6

6

7

8

9

9

10

10

10

10

11

\subsection{Accelerators}

3.3 Polarized beam

3.5 Slow helicity reversals

3.6 Targets

3.7 Spectrometers

3.8 Møller spectrometers

3.9 Detectors

3.9.2 Counting detectors

3.10 Electronics

3.11 Polarimetry

$3.12 Q^{2}$ calibration

4 Results and implications

4.1 Strange form factors

4.2 Electroweak tests
3.4 Helicity-correlated changes in the beam

3.7.1 JLab high-resolution spectrometer

3.7.2 G0 toroidal spectrometer

3.7.3 Crystal spectrometer at Mainz

3.7.4 The Qweak spectrometer

3.7.5 JLab SoLID spectrometer

3.9.1 Integrating detectors

3.11.1 Møller polarimeters

3.11.2 Compton polarimeters

4.2.1 Limits on compositeness

4.2.2 Leptophobic $Z^{\prime}$ boson

4.2.3 Dark light

4.2.4 SUSY 
4.3 Radius of neutron distributions

5 Summary and conclusions

Acknowledgements

References

\section{Introduction}

Electron scattering has proven to be an important tool for exploring the structure of nuclei and the nucleons from which they are made. Highlights include the determination of the precise sizes and shapes of many different nuclei [1] and the discovery that nucleons are composed of electrically charged point-like particles, which are now known as quarks $[2,3]$.

Ultimately, electron scattering from nuclei is due to the electromagnetic interaction of the electrons with the quarks: up quarks with a charge of $2 / 3$ and down quarks with charge $-1 / 3$ (as well as strange quarks). The electromagnetic interaction is relatively weak and can be described accurately as the exchange of a single photon. Consequently, electron scattering data are straightforward to interpret theoretically.

In this review, we describe the field of parity violation in electron scattering (PVES), in which the quantity measured is

$$
A_{P V}=-A_{L R}=\frac{\sigma_{R}-\sigma_{L}}{\sigma_{R}+\sigma_{L}},
$$

where $\sigma_{R}\left(\sigma_{L}\right)$ is the cross section for the scattering of electrons with right (left) helicity. Because electromagnetism conserves parity, any nonzero value of $A_{P V}$ must be due to the weak interaction through exchange of the $\mathrm{Z}$ boson in the Standard Model (SM) or to new physics beyond the SM (BSM). Measuring an asymmetry has a number of advantages, including the cancellation of many possible theoretical and experimental uncertainties.

There are three motivations for this program:

1) To measure the parameters of the SM, especially the couplings of the $\mathrm{Z}$ boson [4];

2) To search for new parity-violating interactions (BSM physics) [5];

3) To measure properties of nuclei that cannot be studied accurately with other probes [6].

The advantages of PVES for the first two points are evident. For studying nuclei, PVES has the advantage that the quarks have different charges when interacting with the $\mathrm{Z}$ boson instead of the photon. For the $\mathrm{Z}$ boson, the charge of the neutron is much larger than that of the proton, in striking contrast to the nucleon charges for photon exchange. Thus, PVES is sensitive to the distribution of the neutrons in a nucleus, in contrast to unpolarized electron scattering, which is sensitive to the distribution of the protons.

\subsection{History of the field}

In the common decays of radioactive nuclei into electrons and neutrinos, weak currents are observed that carry charge. In 1958, Wu et al. [7] found that these charged weak currents violated parity. In the 1970s, neutrinos were observed to interact with matter without producing electrons or muons, proving that there are also neutral weak currents. One of the theories at the time, now called the Standard Model, predicted that the neutral current would also violate parity. However, published experiments on atomic parity violation (APV) suggested that parity violation was much smaller than predicted by the SM. In 1978, the first observation of parity violation in electron scattering was published by Prescott et al. [8, 9] and helped lead to a general acceptance of the SM. These observations marked the beginning of the field of PVES.

The Prescott experiment was challenging because the asymmetry measured was only $10^{-4}$, a tiny fraction of the cross section. To achieve this feat, the experiment required a number of experimental innovations, many of which are still central to the field of PVES today. Perhaps the most important is the development of a polarized electron source based on photoemission from GaAs. The source featured high intensity and the ability to reverse the helicity of the beam without making large changes in the beam properties such as the position, angle, or energy. To verify that the beam was almost perfectly reversed, a set of precision beam monitors was developed. Finally, the signals from the scattering events were integrated rather than counted, as was the practice for virtually all particle physics experiments. Integrating the signals allowed accumulation of the large statistics necessary for measuring the small parity-violating asymmetry.

The Prescott experiment was followed by two PVES experiments using nuclei as targets. Both experiments $[10,11]$, which were designed to test the predictions of the SM, were able to measure even smaller asymmetries. In addition to confirming the predictions of the SM, these efforts demonstrated the power of PVES as a general tool. We will refer to these experiments as Generation I PVES experiments.

The advent of the "spin crisis" in 1989 [12, 13], in which data on the spin-structure functions of the proton indicated that the spin of the proton was not simply the sum of the spins of the valence quarks, raised 
the question of the role of strange quarks in the nucleon [14]. McKeown [15] explained that PVES was a practical way to measure the strangeness content of nucleon elastic form factors. Four experimental programs in three laboratories, the Generation II PVES experiments, followed. The experiments were SAMPLE at the MIT Bates laboratory [16-18], the HAPPEX program at JLab [1921], G0 at JLab [22, 23], and the A4 program at Mainz [24-26]. By the time these programs were well underway, PVES had become accepted as a standard technique in electromagnetic facilities.

The success of the strange-form-factor program led to the publication of some even more challenging experiments providing improved SM tests, SLAC E158 [27] and Qweak [28] at JLab and also the JLab PREx experiment [29], which measured PVES on ${ }^{208} \mathrm{~Pb}$ to determine the charge radius of the neutron distribution. These, along with the future PREx-II and CREx measurements, are the Generation III PVES experiments.

A number of even more challenging Generation IV PVES experiments have been proposed, of which the P2 experiment at Mainz [30] and the MOLLER [31] and SoLID experiments at JLab have been approved. These experiments will push the limits regarding both how small an asymmetry can be measured and how precisely that asymmetry can be measured. The precision of the various PVES experiments is summarized in Fig. 1.

\subsection{Experimental overview}

In a typical PVES experiment, a beam of electrons with

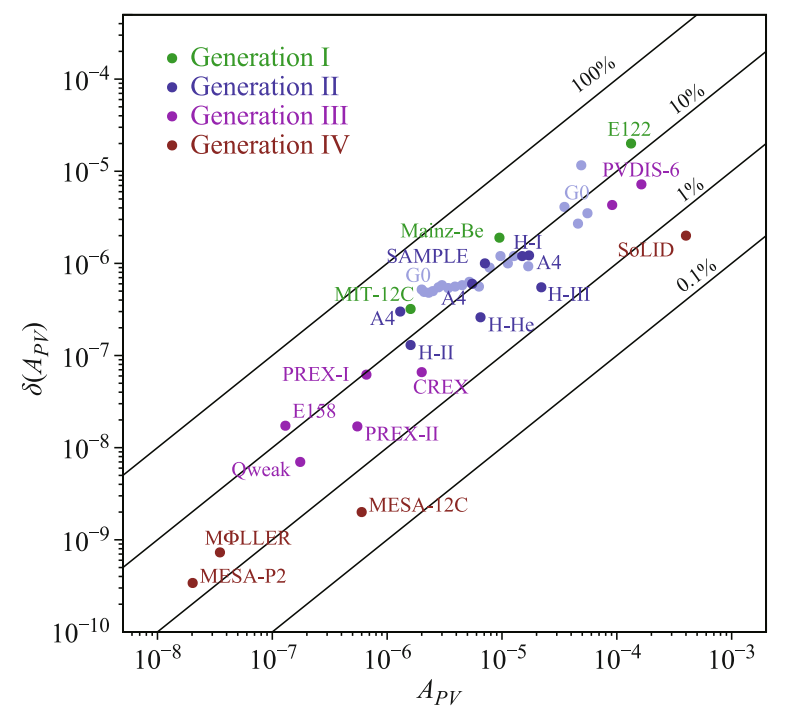

Fig. 1 Summary of past and future PVES measurements. Horizontal axis: measured value of the asymmetry $A_{P V}$. Vertical axis: total experimental error. The diagonal lines indicate the fractional error in the asymmetry measurements. energy $E$ scatters from the target at an angle $\theta$ and emerges with energy $E^{\prime}$. No other particles are detected; the process is called inclusive scattering. The cross section and asymmetry depend mainly on two parameters, the magnitude of the four-momentum transfer,

$$
Q^{2}=4 E E^{\prime} \sin ^{2} \theta / 2
$$

and the electron energy loss $\nu$,

$$
\nu=E-E^{\prime} .
$$

For elastic scattering events, in which no other particles are produced,

$$
Q^{2}=2 M \nu,
$$

where $M$ is the mass of the target. Inelastic events can be identified by the fact that $E^{\prime}$ is less than that predicted by Eq. (4).

The quantity $A_{P V}$ is typically less that $10^{-4} Q^{2}$ $(\mathrm{GeV} / \mathrm{c})^{-2}$, where $Q^{2}$ for PVES experiments ranges from 0.01 to $10(\mathrm{GeV} / \mathrm{c})^{2}$. For lower $Q^{2}$ values, the measured asymmetries are quite small, often well below 1 part per million (ppm), and very high rates are required to make measurements with sufficient statistics. In addition, great care must be taken when reversing the helicity to preserve the properties of the beam. Any systematic difference in the position, angle, or energy of beams of different helicity can cause a difference in the measured rate that has nothing to do with parity violation. Fortunately, the helicity of electron beams can be rapidly and cleanly reversed, typically at rates greater than $1 \mathrm{kHz}$. Slow drifts in the efficiency of the apparatus are thus eliminated, and many systematic errors that are important for cross section measurements, such as the target thickness and angular acceptance of the scattered electron, cancel in the asymmetry. A remarkable precision can be achieved in PVES experiments; sensitivities as small as 20 parts per billion (ppb) have been published, and planned experiments are expected to achieve sensitivities below the ppb level.

Elastic scattering is ideal for PVES experiments. At low $Q^{2}$ values, the cross section is large, so large statistics can be obtained. In addition, only the highest-energy electrons represent elastic scattering. These electrons can be cleanly identified using a magnetic spectrometer or calorimeter and distinguished from the lower-energy electrons from inelastic background processes.

The experimental facilities designed to measure electromagnetic cross sections were also found to be suitable for PVES experiments, even though the data rates for the two types of experiments differ by many orders of magnitude. The experiments are compatible because important cross sections for some scattering angles are 
highly suppressed by the form factors (see the next section), and the facilities were designed to measure these extremely small cross sections. When the same apparatus is used for reactions with large cross sections, the statistics required for PVES become available. However, new detector and data acquisition (DAQ) systems are required to handle the higher rates.

\subsection{Neutrino scattering and PVES}

Neutrino scattering provides an alternative method of measuring the interaction of the $Z$ boson with electrons or nuclei. There are a number of important differences between the two processes, both theoretical and experimental:

1) The neutrino has no vector charge.

2) Neutrino scattering has contributions from terms with both $g^{t}$ and $g^{b}$ purely axial. These terms do not contribute to $A_{P V}$.

3) The radiative corrections are different and in some cases are smaller for neutrinos than for electrons.

4) The energy of the incoming neutrino beam cannot be precisely known, and the scattered neutrino cannot be detected. This reduces the knowledge of individual events. Thus, the data are usually summed over large bins in $Q^{2}$ and $\nu$, washing out some of the important physics.

An extensive literature on this subject exists [32]. One neutrino experiment, however, is especially relevant for PVES. The NuTeV collaboration [33] published a result based on deep inelastic scattering (DIS) with neutrinos that disagrees with the SM. There is an ongoing debate about the interpretation of this result.

\subsection{Atomic parity violation}

A probe complementary to PVES is APV. A parityviolating interaction between the electrons and nucleus in an atom can allow atomic transitions that otherwise are forbidden. A particularly precise experiment on APV in Cs has been published [34]. (The interpretation of that result depends on calculations of the atomic wave functions that have improved since the publication of the result, as discussed in Ref. [4].)

Even more precise APV experiments may be feasible in the future, for example, with trapped Ra ions [35]. The information obtained by APV is very similar to that obtained by elastic scattering from light $N+Z$ nuclei at low $Q^{2}$.

\subsection{Theory of parity violation in potential scattering}

To explain the main features of PVES, we will describe a case that is easy to calculate, namely, nonrelativistic potential scattering [36]. Let a spherical nucleus be composed of $N$ charged objects that have a number density distribution $\rho(r)$. The distribution $\rho(r)$ can be either a classical charge distribution or, for a realistic nucleus, a quantum mechanical probability distribution. The Fourier transform of the number distribution, which is called the form factor, is defined as

$$
F\left(Q^{2}\right) \equiv \int \rho(r) \exp (\mathrm{i} \boldsymbol{q} \cdot \boldsymbol{r}) \mathrm{d}^{3} r,
$$

where $\boldsymbol{q}$ is the momentum transfer, which is the difference between the momenta of the incident and scattered electrons. Clearly

$$
F(0)=\int \rho(r) \mathrm{d}^{3} r=N .
$$

The potential for weak or electromagnetic scattering can be expressed by the formula

$$
V(\mathbf{r})=k e^{2} g^{b} g^{t} \frac{\exp (-M r)}{4 \pi r},
$$

where $M$ is the mass of the exchanged particle, $k$ is a coupling constant, and $g^{b}$ and $g^{t}$ are the charges of the beam and target particles, respectively, in units of $e$. For electromagnetic electron scattering, which involves photon exchange, $M=0, k=1, g^{t}=q_{E M}^{t}$, and $g^{b}=-1$. For the weak interaction, which involves $Z$ exchange, $M=M_{Z}=91 \mathrm{GeV}$, and $k=k_{Z}=\left(\sin \theta_{W} \cos \theta_{W}\right)^{-2}$. Here $e^{2}=4 \pi \alpha$.

For $Z$ exchange, the couplings of $g^{b}$ and $g^{t}$ depend on the helicity of the particle, resulting in the parityviolating asymmetry $A_{P V}$. Values of the helicity difference in $g^{b}$ for $Z$ exchange $\left(g_{A} \equiv g_{L}-g_{R}\right)$ are given in Table 1. For quarks in a nucleus with no angular momentum, the scattering depends only on the average coupling $g_{V}^{t} \equiv\left(g_{R}^{t}+g_{L}^{t}\right) / 2$. However, for polarized electrons, the coupling is different for different helicities. The weak charges are also given in Table 1.

The scattering amplitude in the Born approximation is

$$
f^{L(R)}\left(Q^{2}\right)=\frac{2 k m \alpha g_{L(R)}^{b}}{Q^{2}+M^{2}} g^{t} F\left(Q^{2}\right),
$$

where $m$ is the mass of the electron. The same form factor arises for both weak and electromagnetic scattering, even though the ranges of the two interactions are totally different. Because both weak and electromagnetic scattering are coherent, the total scattering amplitude is 
Table 1 Weak and electromagnetic charges for electrons, light quarks, and the nucleons in units of the electron charge $e$.

\begin{tabular}{cccc}
\hline Particle & $q_{E M}$ & $g_{V}$ & $g_{A}$ \\
\hline$e^{-}$ & -1 & $-\frac{1}{2}+2 \sin ^{2} \theta_{W}$ & $\frac{1}{2}$ \\
$u$ & $\frac{2}{3}$ & $\frac{1}{2}-\frac{4}{3} \sin ^{2} \theta_{W}$ & $-\frac{1}{2}$ \\
$d, s$ & $-\frac{1}{3}$ & $-\frac{1}{2}+\frac{2}{3} \sin ^{2} \theta_{W}$ & $\frac{1}{2}$ \\
$p$ & 1 & $\frac{1}{2}-2 \sin ^{2} \theta_{W}$ & $-\frac{1}{2}$ \\
$n$ & 0 & $-\frac{1}{2}$ & $\frac{1}{2}$ \\
\hline
\end{tabular}

$$
\begin{aligned}
f_{\text {Total }}^{L(R)}\left(Q^{2}\right) & =f_{\gamma}+f_{Z}^{L(R)} \\
& =\left(-\frac{q_{E M}^{t}}{Q^{2}}+\frac{k_{Z} g_{L(R)}^{b} g_{V}^{t}}{M_{Z}^{2}}\right) 2 m \alpha F\left(Q^{2}\right) .
\end{aligned}
$$

The scattering cross section is proportional to the square of the amplitude and hence proportional to $F\left(Q^{2}\right)^{2}$. The sizes and shapes of nuclei have been determined by measuring the cross section, and hence $F\left(Q^{2}\right)$, over a broad range of $Q^{2}$ and inverting the Fourier transform of Eq. (5). Because $M_{Z}^{2} \gg Q^{2}$, the $f_{\gamma}$ term dominates the denominator of $A_{P V}$ but is canceled in the numerator, where the interference term $f_{\gamma} f_{Z}$ dominates.

Thus,

$$
A_{P V}=-\frac{f_{\gamma}\left(f_{Z}^{R}-f_{Z}^{L}\right)}{f_{\gamma}^{2}}=\frac{f_{Z}^{L}-f_{Z}^{R}}{f_{\gamma}}=\frac{Q^{2} k_{Z} g_{A}^{b} g_{V}^{t}}{q_{E M}^{t} M_{Z}^{2}} .
$$

The result is quite remarkable. The form factor is canceled, and $A_{L R}$ is linear in $Q^{2}$, with a slope that depends only on the fundamental parameters of the SM. This follows from Eq. (7). The scattering amplitude is proportional to $F\left(Q^{2}\right)$, regardless of the value of $M$ or the charges.

\subsection{More realistic cases}

The results can easily be extended to the more realistic case where the nucleus comprises up, down, and possibly even strange quarks. There is a charge distribution $\rho^{i}$ for each type, or flavor, of quark and a corresponding flavor form factor $F^{i}\left(Q^{2}\right)$. The nucleus also has antiquarks having the opposite charge, so $\rho^{i}(r)$ is actually the difference in density between the quarks and antiquarks of flavor $i$.

Following Eq. (5), we define the flavor form factors as

$$
F^{i}\left(Q^{2}\right)=\int \rho^{i}(r) \exp (\mathbf{i} \boldsymbol{q} \cdot \boldsymbol{r}) \mathrm{d}^{3} r,
$$

and the quantity $g^{t} F\left(Q^{2}\right)$ in Eq. (7) should be replaced by

$$
F_{Z}\left(Q^{2}\right)=\sum_{i=u, d, s} g_{V}^{i} F^{i}\left(Q^{2}\right)
$$

$$
F_{\gamma}\left(Q^{2}\right)=\sum_{i=u, d, s} q_{E M}^{i} F^{i}\left(Q^{2}\right)
$$

for the weak and electromagnetic amplitudes, respectively. The form factors no longer automatically cancel in $A_{P V}$.

For nuclei with $N=Z$, charge symmetry predicts that $\rho^{u}=\rho^{d}$ and therefore $F^{u}\left(Q^{2}\right)=F^{d}\left(Q^{2}\right) \equiv F\left(Q^{2}\right)$. Then

$$
F_{\gamma}=\frac{1}{3}\left(F-F^{s}\right) ; \quad F_{Z}=-\sin ^{2} \theta_{W} F_{\gamma}-\frac{1}{4} F^{s} .
$$

Finally,

$$
\begin{aligned}
A_{P V} & =\frac{k_{Z} Q^{2}}{2 M_{Z}^{2}} \frac{\sum_{i} g_{V}^{i} F^{i}\left(Q^{2}\right)}{\sum_{i} q^{i} F^{i}\left(Q^{2}\right)} \\
& \rightarrow \frac{G_{F} Q^{2}}{\pi \alpha \sqrt{2}}\left(\sin ^{2} \theta_{W}+\frac{F_{s}}{4 F_{\gamma}}\right),
\end{aligned}
$$

where for the last step we have again assumed charge symmetry in the form $F_{u}\left(Q^{2}\right)=F_{d}\left(Q^{2}\right)$. We have also used the identity $\left(M_{Z} \cos \theta_{W} \sin \theta_{W}\right)^{-2}=\sqrt{2} G_{F} /(\pi \alpha)$ from the SM.

From Eq. (6),

$$
F_{\gamma}(0)=3 Z ; \quad F^{s}(0)=0,
$$

where the last relation holds because the number of strange quarks is the same as the number of strange antiquarks in a nucleus; the net strangeness of a nucleus is zero. The density $\rho^{s}(r)$ can be nonzero if the spatial distributions of the quarks and antiquarks differ. Thus, at low $Q^{2}, F_{s}$ is expected to be small, and the form factors now cancel, so a measurement of $A_{P V}$ can precisely test the SM [37]. For light nuclei at larger $Q^{2}, A_{L R}$ provides a measurement of $F_{s}$.

For heavy nuclei, especially where $N \neq Z$, the spatial distribution of neutrons is expected to be larger than that of protons, so $F_{u}\left(Q^{2}\right) \neq F_{d}\left(Q^{2}\right)$ [38]. This important effect can be measured using PVES, as discussed in Section 2.5.

The above discussion applies to nonrelativistic particles. An interesting new phenomenon does arise in relativistic electron scattering. The helicity of the electron is preserved in the scattering, so the angular momentum changes by one unit for backscattering. For elastic scattering from a spinless target, angular momentum cannot be absorbed by the nucleus, so backscattering is forbidden. The modifications to the cross section when it is calculated with a full relativistic theory do not change $A_{P V}$ :

$$
\frac{\mathrm{d} \sigma}{\mathrm{d} \Omega}=\frac{\left(\alpha g^{b} g^{t}\right)^{2}}{4 E^{2} \sin ^{4} \frac{\theta}{2}} F^{2}\left(Q^{2}\right) \rightarrow \frac{\left(\alpha g^{b} g^{t}\right)^{2}}{4 E^{2} \sin ^{4} \frac{\theta}{2}} F^{2}\left(Q^{2}\right) \cos ^{2} \frac{\theta}{2},
$$

where the expression after the arrow is correct for the rel- 
ativistic case. The only change is the factor of $\cos ^{2} \theta / 2$.

For scattering from a target with spin, such as the proton, backscattering is allowed, and the spin of the target is flipped. Targets with spin also have magnetic moments, and the resulting magnetic fields contribute appreciably to the scattering, especially at large angles. In addition, backscattered electrons can scatter only from quarks with opposite helicity in the center-of-mass (CM) frame. Thus, in this case the axial charge of the quarks contributes to the asymmetry. These features appear in the detailed formulae in Section 2.

\subsection{Precision of SM predictions}

One of the beauties of the SM is that it makes many predictions with spectacular precision. The SM requires only three parameters as input; the typical choice is $\alpha$, $G_{F}$ from muon decay, and $M_{Z}$ from collider experiments. These parameters are now known to very high accuracy. The SM is in fact a perturbation theory with a small coupling constant $\alpha / 2 \pi \sim 10^{-3}$; hence, the first-order approximations, called tree-level predictions, are expected to be accurate to the $0.1 \%$ level. Furthermore, the SM is a renormalizable theory. This means that, in principle, these higher-order corrections can be made to any order and with arbitrary precision.

\subsubsection{Higher-order corrections}

One problem with the SM is that the higher-order corrections, called radiative corrections, are in fact much larger than would be expected on the basis of the small coupling constant. There are two main reasons for this.

The first arises from the fact that the SM unifies physics over a striking breadth of the energy scale. Hadrons in the theory have structure at the $100 \mathrm{MeV}$ scale, whereas the mass of the $Z$ is nearly $100 \mathrm{GeV}$. A quark isodoublet, the $b$ and $t$ quarks, has a mass difference well in excess of $100 \mathrm{GeV}$. Recent precise measurements of the top quark mass and also the discovery of the Higgs particle and determination of its mass have further reduced the uncertainties in the radiative corrections. These large energy differences give large logarithms that enhance the radiative corrections from the naive level of $0.1 \%$ to about $5 \%$. (The precise calculation of these radiative corrections requires knowledge of the quark masses and the Higgs mass, in addition to the three parameters mentioned above.) The second feature that makes the radiative corrections large is that although the vector charges of unit charge particles are much smaller than the axial charges, radiative correction with a vector charge often involves an axial charge, thus

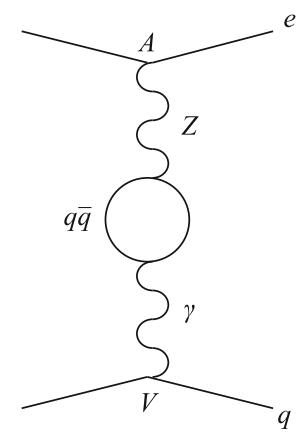

Fig. 2 Radiative correction that reduces $A_{P V}$ for $Q_{W}(p)$ and $Q_{W}(e)$. For $Q_{W}(e)$ the bottom line also denotes an electron.

enhancing the correction from $\sim 5 \%$ to $\sim 50 \%$.

\subsubsection{Uncertainties in radiative corrections}

Radiative corrections include contributions from light quarks. Light quarks have strong interactions, and uncertainties in the effects of these strong interactions introduce uncertainties in the effects of the weak interactions [39]. The size of these uncertainties varies from negligible up to about $100 \%$, depending on the process. A typical radiative correction is the vacuum polarization diagram shown in Fig. 2, where the vector coupling is now with the fractionally charged quarks in the loop, and the $1-4 \sin ^{2} \theta_{W}$ factor is absent. In forward scattering from nuclei, the corrections are small and well known. In backscattering from the proton, where significant radiative corrections can involve two different quarks in the proton, the uncertainties are among the highest.

\subsubsection{Experimental implications}

Processes in which the SM predictions are very reliable are especially suitable for precise experimental studies. A classic example is the measurement of the radius $R_{n}$ of the distribution of neutrons in a heavy nucleus. There are many possible ways to measure this quantity, such as proton, $\alpha$ particle, or pion scattering, antiproton X-rays, and photoproduction of neutral pions. For all of these methods, the main uncertainty in extracting $R_{n}$ is the lack of understanding of the strong interactions of the probes. With PVES, the theoretical errors are negligible because the electron is used as the probe.

\section{Theory}

In this section, we discuss in more detail the general theory used to interpret PVES experiments. In later subsubsections, we explain the formulae appropriate for specific experiments. 


\subsection{Kinematics}

First, we need to introduce kinematic variables in addition to those given in Eqs. (2) and (3). The recoiling target may be left in an excited state characterized by a mass $W$, which is given by

$$
W^{2}=2 M \nu-Q^{2} .
$$

Elastic scattering is characterized by $W=M$, and then the variable

$$
x \equiv \frac{Q^{2}}{2 M \nu}
$$

is equal to 1 from Eq. (4). For low $Q^{2}, \nu \approx 0$, and in this limit $Q^{2}$ is the same as the $Q^{2}$ defined in Section 2.1.

For any inelastic scattering from the proton,

$$
W \geqslant M_{p}+m_{\pi^{0}},
$$

where $m_{\pi^{0}}$ is the mass of the lowest-energy particle that can be produced, the $\pi^{0}$. To identify elastic scattering, the apparatus must have sufficient resolution in both $E^{\prime}$ and $\theta$ to distinguish $W=M_{p}$ from $W=M_{p}+m_{\pi^{0}}$.

To identify elastic scattering from a nucleus, any event with $W>M+\Delta$ must be rejected (where $\Delta$ is the energy of the lowest excited state). This can be achieved for selected nuclei with $\Delta$ on the order of a few megaelectron volts by using an apparatus with high energy resolution.

In Section 1.6, we noted that for potential scattering at backward angles, the axial charges of the quarks contribute to parity violation. However, in heavily relativistic cases, the angle is a poor measure of the effect. Instead, the relevant variable is $\varepsilon=[1+$ $\left.\left.2(1+\tau) \tan ^{2} \theta / 2\right)\right]^{-1}$. The contribution from axial target charges is suppressed by the kinematics when $\varepsilon$ is near unity. Experiments aimed at studying the axial charge must have $\varepsilon \sim 0$.

The same variables are useful for DIS, which is elastic scattering from individual quarks in the nucleon. Here the variable $x$ is the fraction of the proton's momentum carried by the struck quark in a frame where the proton momentum is large. Another important variable, especially in DIS, is

$$
y=\nu / E,
$$

which plays a role similar to that $\varepsilon$ plays in elastic scattering. For large $y$, the axial charges of the quarks contribute to $A_{P V}$.

The variable $y$ is also useful for describing elastic scattering, especially for Møller scattering. Here, the maximum $Q^{2}$ value always corresponds to $y=1 / 2$. In addition,

$$
y=\sin ^{2} \frac{\theta_{C M}}{2}
$$

and

$$
\theta_{L}=\sqrt{\frac{2 m(1-y)}{E y}},
$$

where $\theta_{C M(L)}$ is the scattering angle in the CM (laboratory) frame. For Møller scattering at high energies, $\theta_{L}$ is quite small.

\subsection{Phenomenology}

An important tool for analyzing PVES experiments at low $Q^{2}$ is the phenomenological four-fermion Lagrangian,

$$
\begin{aligned}
\mathcal{L}_{\mathrm{PV}}^{e f} & =\frac{G_{F}}{\sqrt{2}} \bar{e} \gamma^{\mu} \gamma^{5} e \sum_{q=u, d, s} C_{1 q} \bar{q} \gamma_{\mu} q \\
+ & \frac{G_{F}}{\sqrt{2}} \bar{e} \gamma^{\mu} e\left(\sum_{q=u, d, s} C_{2 q} \bar{e} \gamma_{\mu} \gamma^{5} q+C_{2 e} \bar{e} \gamma_{\mu} \gamma^{5} e\right),
\end{aligned}
$$

where $C$ represents independent coupling constants, and $G_{F}$ is the Fermi constant. $\mathcal{L}_{\mathrm{PV}}^{e f}$ is the most general form possible for any physics with a distance scale much shorter than that corresponding to the $Q^{2}$ of the experiments. This Lagrangian is useful for both SM and BSM physics.

In the SM, where parity violation arises from the exchange of a single $Z$ boson, the $C$ values are the products of the weak charges given in Table 1. For example,

$$
C_{1 q}=2 g_{e}^{A} g_{q}^{V} .
$$

Further, in this case the weak charges can be determined from neutrino scattering and used to predict $A_{P V}$. However, for many examples of BSM physics, the $C$ values cannot be expressed as products of charges and cannot be determined from other low-energy experiments.

Measuring each of the $C$ values independently and as precisely as possible is a good way to search for BSM physics. The other implication of the generality of $\mathcal{L}_{\mathrm{PV}}^{e f}$ is that the five $C$ values are the only parameters related to short-range BSM physics that can be measured using PVES.

\subsection{Weak charges}

In PVES experiments with nuclei, a weak charge $Q_{W}$ is defined as a combination of the $C_{1 i}$ :

$$
\begin{aligned}
& Q_{W}(Z, N)=-2\left[C_{1 u}(2 Z+N)+C_{1 d}(Z+2 N)\right] \\
& =Z\left(1-4 \sin ^{2} \theta_{W}\right)-N .
\end{aligned}
$$

In the SM, $Q_{W}$ is the vector charge of the nucleus times 
Table 2 Values for the coupling constants with radiative corrections in the SM. Here $\rho_{e}^{\prime}=0.9887, \rho_{e}=1.0007, \hat{\kappa}_{e}^{\prime}=1.0038, \hat{\kappa}=$ 1.0297 , and $\sin ^{2} \theta_{W}=0.2312$. Without radiative corrections, the $\rho$ ' and $\hat{\kappa}$ 's are unity.

\begin{tabular}{cc}
\hline Constant & SM expression \\
\hline$C_{1 u}$ & $\rho_{e}^{\prime}\left(-\frac{1}{2}+\frac{4}{3} \hat{\kappa}_{e}^{\prime} \sin ^{2} \theta_{W}\right)$ \\
$C_{1 d, s}$ & $\rho_{e}^{\prime}\left(\frac{1}{2}-\frac{2}{3} \hat{\kappa}_{e}^{\prime} \sin ^{2} \theta_{W}\right)$ \\
$C_{2 u}$ & $\rho_{e}\left(-\frac{1}{2}+2 \hat{\kappa}_{e} \sin ^{2} \theta_{W}\right)$ \\
$C_{2 d, s}$ & $\rho_{e}\left(\frac{1}{2}-2 \hat{\kappa}_{e} \sin ^{2} \theta_{W}\right)$ \\
$C_{2 e}$ & $\rho_{e}\left(\frac{1}{2}-2 \hat{\kappa}_{e} \sin ^{2} \theta_{W}\right)$ \\
\hline
\end{tabular}

the axial charge of the electron. In Møller scattering, the weak charge is

$$
Q_{W}(e)=-2 C_{2 e} .
$$

The definition of $Q_{W}$ applies in any theory, but calling it a charge makes sense only for theories where Eq. (15) holds.

Because the Lagrangian $\mathcal{L}_{\mathrm{NC}}^{e f}$ is not renormalizable, it is not possible to know all of the higher-order corrections. However, because the SM is renormalizable, the approach implemented in the field is to use the radiative corrections calculated with the SM to adjust the definitions of the coupling constants, as is done in Table 2. Except for the most precise experiments, the modified $\mathcal{L}_{\mathrm{PV}}^{e f}$ without additional radiative corrections can describe the data well, and the remaining small correction can be applied.

For the weak charges of the proton and electron, the corrections are large. Neglecting radiative corrections, $Q_{W}(p)=-Q_{W}(e)=0.075$, whereas with the corrections, $Q_{W}(p)=0.071$ and $Q_{W}(e)=-0.045$ [40]. The diagram in Fig. 2 decreases both weak charges, but the $W W$ box diagram in Fig. 3 applies only to the proton. The remarkable feature is that even though the weak charges of the proton and electron are "really" equal and opposite, at low energy, these particles behave as if the magnitudes of their charges were quite different [4].

\subsection{Strange form factors}

Scattering from a nucleon is more complex than potential scattering for two reasons:

1) The nucleon has a magnetic moment, which makes a large contribution, especially at larger values of $Q^{2}$.

2) The proton is light enough that it has significant recoil during scattering.

With the contribution of the magnetic moment, the cross section now depends on two Sachs form factors,

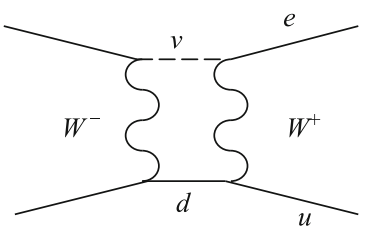

Fig. 3 Radiative correction that increases $A_{P V}$ for $Q_{W}(p)$ but has no effect on $Q_{W}(e)$ because there is no doubly charged electron.

the electric, $G_{E}\left(Q^{2}\right)$, and the magnetic, $G_{M}\left(Q^{2}\right)$, and is given by

$$
\frac{\mathrm{d} \sigma}{\mathrm{d} \Omega}=\left.\frac{\mathrm{d} \sigma}{\mathrm{d} \Omega}\right|_{\mathrm{Mott}} \frac{1}{\varepsilon(1+\tau)}\left[\varepsilon G_{E}^{2}\left(Q^{2}\right)+\tau G_{M}^{2}\left(Q^{2}\right)\right],
$$

where $\tau=Q^{2} /\left(4 M_{p}\right)^{2}$. At low $Q^{2}$, where the recoil effects are small, $G_{E} \rightarrow F\left(Q^{2}\right)$. In addition, at low $Q^{2}$, $G_{E}^{p}=1$ and $G_{M}^{p}=\mu_{p}$, where $\mu_{p}$ is the magnetic moment of the proton. The form factors are observed to fall with $Q^{2}$. Even at this stage, it can be seen that the form factors are unlikely to cancel in $A P V$.

The nucleon form factors for both the proton and neutron can be decomposed in terms of the quark form factors $G_{E}^{i}$ and $G_{M}^{i}$ as was done for the nucleon form factors in Eq. (10). For example,

$$
G_{E}^{p}=\sum_{i} q^{i} G_{E}^{i}=\frac{2}{3} G_{E}^{u}-\frac{1}{3} G_{E}^{d}-\frac{1}{3} G_{E}^{s} .
$$

If we assume charge symmetry, then $G_{E}^{u}$ for the proton equals $G_{E}^{d}$ for the neutron, and so on. The strange form factor is the same for the proton and for the neutron. Then

$$
G_{E}^{n}=\frac{2}{3} G_{E}^{d}-\frac{1}{3} G_{E}^{u}-\frac{1}{3} G_{E}^{s} .
$$

The weak nucleon electric form factors are obtained from the quark form factors by using the weak charges. The same is true for the magnetic form factors.

Thus, for the nucleon, there are three independent electric and three independent magnetic form factors. Because the proton and neutron form factors have been measured, a convenient set of form factors is $G_{E}^{p}, G_{E}^{n}$, and $G_{E}^{s}$. For the same reason that $F^{s}(0)=0$, as discussed in Section 1.6, $G_{E}^{s}(0)=0$. The contribution of the strange quarks to the static magnetic moment may be nonzero, so it is possible that $G_{M}^{s}(0) \equiv \mu_{s} \neq 0$.

The asymmetry for the proton is

$$
A_{P V}=\frac{G_{F} Q^{2}}{\pi \alpha \sqrt{2}}\left(Q_{W}^{p}+A_{M}+A_{s}+A_{A}\right),
$$

where

$$
A_{M}=\frac{\varepsilon G_{E}^{p} G_{E}^{n}+\tau G_{M}^{p} G_{M}^{n}}{\varepsilon\left(G_{E}^{p}\right)^{2}+\tau\left(G_{M}^{p}\right)^{2}}
$$




$$
\begin{aligned}
A_{s} & =\frac{\varepsilon G_{E}^{p} G_{E}^{s}+\tau G_{M}^{p} G_{M}^{s}}{\varepsilon\left(G_{E}^{p}\right)^{2}+\tau\left(G_{M}^{p}\right)^{2}} \\
A_{A} & =\frac{\left(1-4 \sin ^{2} \theta_{W}\right) \sqrt{1-\varepsilon} \sqrt{\tau(1+\tau)} G_{M}^{p} \tilde{G}_{A}^{p}}{\varepsilon\left(G_{E}^{p}\right)^{2}+\tau\left(G_{M}^{p}\right)^{2}} .
\end{aligned}
$$

There is an additional form factor, $\tilde{G}_{A}^{p}$. It arises from the axial charge of the nucleon, which has no effect in electromagnetic scattering. The radiative corrections to this term are large, possibly $\sim 100 \%$, because of strong interaction effects that cannot be precisely calculated [41]. Therefore, it must be treated as an unknown, in addition to $G_{E}^{s}$ and $G_{M}^{s}$, when PVES data are interpreted. All of the other quantities, in particular the electromagnetic form factors, have been measured in electron scattering experiments.

It is interesting that for experiments with moderate values of $Q^{2}$ (between 0.1 and $\left.1.0(\mathrm{GeV} / \mathrm{c})^{2}\right)$, the largest contribution to the asymmetry is from the $G_{M}^{p} G_{M}^{n}$ component of $A_{M}$. This is because the $Z$ boson interacts with the magnetic moment of the proton. The contribution from this term must be subtracted to obtain the strange form factors.

Depending on the kinematics, the asymmetry is sensitive to different terms. At forward angles, where $\varepsilon$ is near unity, the troublesome $A_{A}$ term is suppressed by the $\sqrt{1-\varepsilon}$ factor. At forward angles and higher $Q^{2}$ values, the $A_{A}$ term is suppressed, and the main contribution to the asymmetry is a linear combination of $G_{E}^{s}$ and $G_{M}^{s}$.

The SAMPLE, G0 [22], and A4 [26] experiments measured the asymmetry in the backward direction with both a hydrogen and a deuterium target. By subtracting the asymmetries, the $A_{A}$ component is canceled, but the $G_{M}^{s}$ contribution remains. Thus, $G_{E}^{s}$ can be isolated from the forward data.

At very low $Q^{2}$, the $A_{M}$ and $A_{s}$ terms become negligible, and $A_{P V}$ is dominated by the first term in Eq. (19), which is proportional to $Q_{W}^{p}$. This is the kinematics explored by the Qweak experiment. (Note that both $G_{E}^{s}$ and $G_{E}^{n}$ are proportional to $Q^{2}$ at low $Q^{2}$.) There is an important radiative correction to this term $[42,43]$ owing to theoretical error introduced by the diagram shown in Fig. 4. The P2 experiment plans to run at lower beam energies, at which this correction is smaller and less uncertain.

Another strategy for isolating $G_{E}^{s}$ and $G_{M}^{s}$, used by the HAPPEX [20] collaboration, is to measure $A_{P V}$ for both hydrogen and the spinless nucleus ${ }^{4} \mathrm{He}$. The asymmetry for a spinless nucleus with $N=Z$ is given by [44]

$$
A_{P V}=\frac{G_{F} Q^{2}}{\pi \alpha \sqrt{2}}\left[\sin ^{2} \theta_{W}+\frac{G_{E}^{s}}{2\left(G_{E}^{\gamma p}+G_{E}^{\gamma n}\right)}\right] .
$$

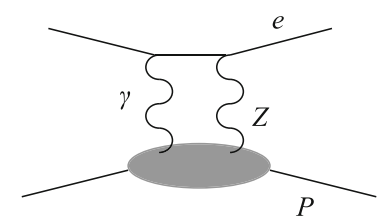

Fig. 4 Gamma- $Z$ box diagram that is important for $Q_{W}(p)$. The blob in the proton line indicates that the photon and $Z$ interact on different quarks in the proton.

This method is quite clean but impractical for $Q^{2}$ values much above $0.1(\mathrm{GeV} / \mathrm{c})^{2}$ because the helium form factor, and thus the event rate, becomes too small.

At lower $Q^{2}$ values, similar to those in the Qweak experiment, the $G_{E}^{s}$ term is negligible, and it is possible to test the SM using Eq. (20). Even accounting for nuclear effects such as isospin mixing and meson-exchange currents, the errors in interpreting the result of a possible ${ }^{12} \mathrm{C}$ experiment as an SM test should be small [37].

\subsection{Radius of neutron distribution}

For a heavy nucleus with $N \neq Z, A_{P V}$ is given by

$$
A_{P V}=\frac{G_{F} Q^{2}}{\pi \alpha \sqrt{2}}\left[\sin ^{2} \theta_{W}-\frac{1}{4}\left(1-\frac{F^{n}\left(Q^{2}\right)}{F^{p}\left(Q^{2}\right)}\right)\right] .
$$

At low $Q^{2}, F^{n} / F^{p}=N / Z$. If the distributions of protons and neutrons in the nucleus differ, $F^{n} / F^{p}$ will be a function of $Q^{2}$ that is sensitive to the difference between the charge radii of protons and neutrons.

Equation (21) is derived assuming that the beam can be described by a plane wave, which is a poor approximation for nuclei with large $Z$. By using the Dirac equation, the asymmetry can be computed reliably [45], and the sensitivity to $A_{P V}$ remains and provides an accurate way to determine $R_{n}[46]$.

\subsection{Parity violation in deep inelastic scattering}

At high $Q^{2}$ values, the cross section for electrons to scatter incoherently from individual quarks, i.e., DIS, is quite large. The process is essentially elastic scattering from a quark. It is the reaction in which parity violation (PVDIS) in neutral currents was observed by Prescott et al. The asymmetry is [47]

$$
A^{P V}=\left(\frac{G_{F} Q^{2}}{4 \sqrt{2} \pi \alpha}\right)\left(Y_{1} a_{1}+Y_{3} a_{3}\right) .
$$

Most of the physics emerges in the Callan-Gross approximation, where

$$
Y_{1}=1 ; \quad Y_{3}=\frac{1-(1-y)^{2}}{1+(1-y)^{2}} .
$$

The quantity $Y_{3}$ is small for small values of $y$, and $Y_{3} \rightarrow 1$ 
as $y \rightarrow 1$. The $a_{i}$ depend on the DIS structure functions $F_{i}$ :

$$
a_{1}=\frac{2 g_{A}^{e} F_{1}^{\gamma Z}}{F_{1}^{\gamma}} ; \quad a_{3}=\frac{g_{V}^{e} F_{3}^{\gamma Z}}{F_{1}^{\gamma}} .
$$

In the quark-parton model (QPM), the structure functions are given in terms of the parton distribution functions (PDFs) $f_{i}(x)$, where $i$ denotes the quark flavor. They are

$$
\begin{aligned}
& F_{1}^{\gamma}=\frac{1}{2} \sum_{i}\left(q_{i}^{e m}\right)^{2}\left(f_{i}(x)+\bar{f}_{i}(x)\right), \\
& F_{1}^{\gamma Z}=\sum_{i} q_{i}^{e m} g_{V}^{i}\left(f_{i}(x)+\bar{f}_{i}(x)\right), \\
& F_{3}^{\gamma Z}=2 \sum_{i} q_{i}^{e m} g_{A}^{i}\left(f_{i}(x)-\bar{f}_{i}(x)\right) .
\end{aligned}
$$

Here the $\bar{f}_{i}(x)$ are the PDFs of the antiquarks. Quarkantiquark pairs including strange quarks dominate at low $x$ and are called "sea" quarks. In the valence region, near $x \approx 0.3, f_{u}^{p} \approx 2 f_{u}^{p}$, consistent with the idea that the proton is made up of two up quarks and one down quark. As $x \rightarrow 1$, some quark models predict that $f_{u}^{p} \neq 2 f_{u}^{p}$ [48].

In the SM, the $a_{1}$ term depends on the $C_{1 i}$, and the $a_{3}$ term depends on the $C_{2 i}$. Because $a_{3}$ is proportional to $g_{V}^{e}$, it is small. To obtain sensitivity to the $a_{3}$ term, the kinematics must be chosen so that $y$, and hence $Y_{3}$, is large. Because DIS is incoherent scattering from isolated quarks, the radiative corrections can be precisely calculated, in contrast to the case for the $A_{A}$ term in elastic scattering.

\subsubsection{PVDIS for deuterium}

For electron-deuteron scattering, charge symmetry implies that for the PDFs,

$$
f_{u}^{p}(x)=f_{d}^{n}(x) ; \quad f_{d}^{p}(x)=f_{u}^{n}(x) .
$$

For large $x$, the contributions from strange quarks are negligible, and the PDFs cancel. Then

$$
a_{1}^{d}=\frac{6}{5}\left(2 C_{1 u}-C_{1 d}\right) ; \quad a_{3}^{d}=\frac{6}{5}\left(2 C_{2 u}-C_{2 d}\right) .
$$

PVDIS on the deuteron has a number of attractive features:

1) The hadronic structure cancels to first order in the asymmetry.

2) The cross section remains large for large values of $Q^{2}$, so large asymmetries can be measured with good statistics.

3) As stated in the previous section, $A_{P V}$ at large values of $y$ is sensitive to the $C_{2 i}$ without the complica- tion of unknown radiative corrections. It is the most favorable process for accessing this information.

Thus, $A_{P V}^{D I S}$ at large $x$ is an ideal candidate for searching for BSM physics.

\subsubsection{Charge symmetry}

It is possible that charge symmetry violation (CSV) occurs [49]; CSV can be characterized by new structure functions,

$$
\delta f_{u}=f_{u}^{p}-f_{d}^{n} ; \quad \delta f_{d}=f_{d}^{p}-f_{u}^{n} .
$$

The fractional change in $a_{1}^{d}$ is

$$
\frac{\delta a_{1}^{C S V}}{a_{1}^{d}}=\left[-\frac{3}{10}+\frac{2\left(2 C_{1 u}+C_{1 d}\right)}{\left(2 C_{1 u}-C_{1 d}\right)}\right]\left(\frac{\delta f_{u}-\delta f_{d}}{f_{u}+f_{d}}\right) .
$$

It is quite possible that the $\delta f$ terms have a different $x$ dependence than the $f$ terms; in this case, the $x$ dependence of $A_{P V}^{D I S}$ would provide a clean CSV signature. $\mathrm{CSV}$ is one of the explanations [50-53] for the difference between the value of $\sin ^{2} \theta_{W}$ measured by the $\mathrm{NuTeV}$ collaboration [33] and the prediction from the SM.

\subsubsection{Higher twist}

In the context of quantum chromodynamics (QCD), the QPM can be extended to become a rigorous theory. With QCD, the PDFs become dependent on $Q^{2}$ in a logarithmic manner and also pick up extra "higher twist" (HT) terms that vary as $1 / Q^{2}$. For $A_{P V}^{D I S}$, the logarithmic factors cancel. Most of the HT terms involve gluons, the particles that strongly bind the quarks in the nucleon. For DIS cross section data at large $x$, these HT terms are quite large. However, as pointed out by Bjorken [54] and Wolfenstein [55], all the HT terms involving gluons cancel in $A_{P V}^{D I S}$. The remaining terms involve quark-quark correlations [56], which are calculated to be small in most models [56-61]. If instead the HT contributions are observed to be significant, our understanding of nucleon structure will change.

\subsubsection{PVDIS with a proton target}

For a proton target, the PDFs do not cancel, and the result is

$$
a_{i}^{p}(x) \approx \frac{3}{4}\left[\frac{6 C_{1 u} u(x)-3 C_{1 d} d(x)}{u(x)+\frac{1}{4} d(x)}\right],
$$

where $u(x) \equiv f_{u}^{p}(x)$, and $d(x) \equiv f_{d}^{p}(x)$. In the SM, 


$$
a_{1}^{p} \sim\left[\frac{1+0.912 d(x) / u(x)}{1+0.25 d(x) / u(x)}\right] .
$$

Measurements of PVDIS with a proton target can determine whether the ratio $d / u$ at large $x$ is 0.5 as predicted by valence models, 0 as in some broken $S U(6)$ models, or somewhere in between [62].

The traditional method of measuring $d / u$ is to compare the DIS cross section of the proton with that of the neutron. The problem is that the neutron cross section is traditionally measured with a deuterium target, but there are large uncertainties at large $x$ arising from the nuclear physics of the neutron. Methods of minimizing the nuclear effects include detecting the slow recoil proton in DIS events and comparing the DIS cross sections of ${ }^{3} \mathrm{He}$ and ${ }^{3} \mathrm{H}$. The advantage of PVDIS is that only a proton target is used, so that nuclear physics corrections are entirely absent. The potential precision from the various methods is compared in Fig. 5 .

\subsection{Møller scattering}

In the SM, the electron is a simple point particle with no unknown structure. Hence, the electron is an ideal target for studying the SM and beyond. The cross section for electron-electron scattering, called Møller scattering, is

$$
\left(\frac{\mathrm{d} \sigma}{\mathrm{d} \Omega}\right)_{C M}=\frac{\alpha^{2}}{4 E_{L a b}}\left[\frac{1+y^{4}+(1-y)^{4}}{y^{2}(1-y)^{2}}\right]
$$

and the asymmetry is [64]

$$
A_{P V}=Q_{W}^{e} \frac{Q^{2} G_{F}}{\sqrt{2} \pi \alpha}\left(\frac{1-y}{1+y^{4}+(1-y)^{4}}\right) .
$$

The expression is quite simple and clearly provides a clean measurement of $Q_{W}^{e}$. The radiative corrections [4], which in Section 2.2 were described as very large, can be calculated quite reliably, because the only hadrons involved are in the loops in the radiative corrections.

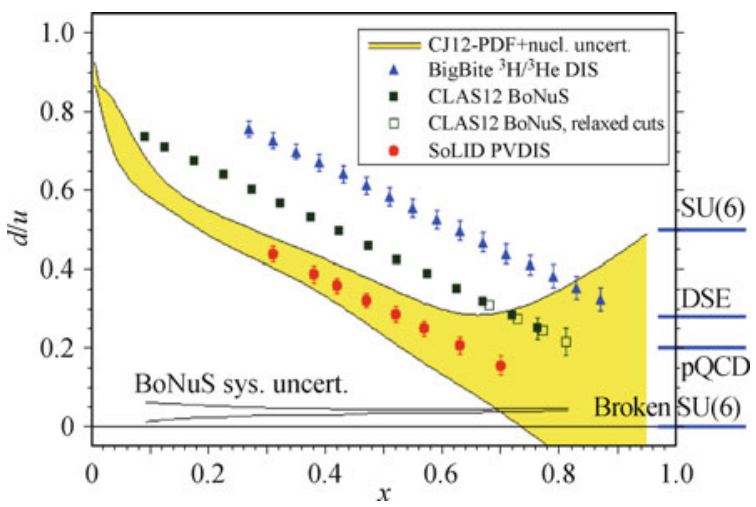

Fig. 5 Anticipated results for various methods for extracting the ratio $d / u$ for the proton. Current knowledge, including uncertainty due to nuclear corrections, is represented by the yellow band [63]
Møller scattering is potentially the most sensitive way to measure $\sin ^{2} \theta_{W}$ at low energies and can even provide results competitive with those of the LEP facility at CERN on the $Z$ pole [4].

\section{Experimental details}

To measure $A_{P V}$, there are two basic requirements. The first is to observe the small asymmetry by collecting sufficient statistics and avoiding systematic effects such as helicity correlations in beam parameters. Once a nonzero asymmetry is observed, the second requirement is to control systematic errors that scale with the asymmetry, such as the measurement of $Q^{2}$ or $P_{e}$. Often the scale errors are required to be well below $5 \%$, in some cases $1 \%$ or even lower.

\subsection{Rates and statistical errors}

For a parity experiment with $N$ events detected, the fractional error in the asymmetry is

$$
\frac{\delta A_{P V}}{A_{P V}}=\frac{1}{P_{e} \sqrt{N}}
$$

where $P_{e}$ is the polarization of the electron beam. The number of events $N$ in an experiment is given by

$$
N=I z n \frac{\mathrm{d} \sigma}{\mathrm{d} \Omega} \Delta \Omega T,
$$

where $I$ is the beam current, $z$ is the target thickness, $n$ is the target density, $\mathrm{d} \sigma / \mathrm{d} \Omega$ is the differential cross section for the process, $\Delta \Omega=\Delta \cos \theta \Delta \phi$ is the angular acceptance of the detector, and $T$ is the running time.

To measure the small PVES asymmetries, each of the factors in Eq. (33) must be as large as possible. Typical beam currents of $50 \mu \mathrm{A}$, and even currents as large as $180 \mu \mathrm{A}$, are available as long as the target can tolerate the beam power. The target thickness $z$ is limited by the fact that electrons radiate when passing through material. Calculations show that once about $10 \%$ of the beam energy is lost to radiation, the rate of useful events no longer increases with $z$. For low-density targets, including the important cases of $\mathrm{H}, \mathrm{D}$, and $\mathrm{He}$, the radiation length criterion allows targets of more than a meter in length, which may not be practical for some experiments. The experiments require extensive beam time to collect the data, with $T$ being on the order of $10^{6}$ to $10^{7} \mathrm{~s}$. For example, the G0 experiment published $700 \mathrm{~h}$ of data, a very typical data set size for a PVES experiment at JLab.

The other parameters in Eq. (33) involve some tradeoffs. For example, the cross section, angular acceptance, 
and asymmetry all depend on the electron scattering angle $\theta$. To keep the asymmetry reasonably constant over the acceptance, usually $\Delta \theta / \sin \theta \equiv b \leqslant 0.3$.

To optimize the statistics for a parity experiment, the kinematics, including the beam energy $E$ and scattering angle $\theta$, must be chosen carefully. Both the cross section and asymmetries depend on these parameters. For the simplest case of elastic scattering from a heavy, spinless, isoscalar nucleus, the asymmetry and cross section are given by

$$
A_{P V}=A_{0} Q^{2} ; \quad \frac{\mathrm{d} \sigma}{\mathrm{d} \Omega}=\frac{E^{2} \cos ^{2}(\theta / 2)|F|^{2}}{Q^{4}} .
$$

With this formula, we define a figure of merit (FOM),

$$
M=\frac{\mathrm{d} \sigma}{\mathrm{d} \Omega} \Delta \Omega A_{P V}^{2} \sim Q^{2}|F|^{2} b \cos ^{2}(\theta / 2) \Delta \phi \sim \frac{1}{T},
$$

which is proportional to the inverse of the running time. The optimum for ${ }^{12} \mathrm{C}$ occurs for $Q^{2} \sim 0.02(\mathrm{GeV} / \mathrm{c})^{2}$ and is independent of the beam energy so long as the desired $Q^{2}$ occurs at forward angles. For elastic scattering from the proton, the form factor falls more slowly at larger $Q^{2}$ values than it does for nuclei, and larger asymmetries are possible. For DIS, the form factor is constant, and the size of the asymmetry is limited only by the beam energy.

\subsection{Accelerators}

An accelerator must be available that provides a beam with sufficient energy to obtain the desired kinematics and an intensity that yields sufficient statistics. For PVES experiments in which counting techniques are used, the duty factor is also important. These quantities for various facilities are summarized in Table 3.

For PVDIS and Møller scattering, higher energies provide larger asymmetries. In addition, for PVDIS, the theoretical uncertainties are smaller at higher energies. The $11 \mathrm{GeV}$ beams available with the JLab upgrade are critical to these experiments. For elastic scattering from nuclei, where the absolute energy resolution is critical for rejecting inelastic events, facilities with lower beam energies are competitive. For elastic scattering from hydrogen, an energy of about $3-4 \mathrm{GeV}$ is ideal, but lower

Table 3 Approximate properties of accelerators used for PVES experiments.

\begin{tabular}{cccc}
\hline Accelerator facility & Energy & Intensity & Duty factor \\
\hline SLAC & $20-50 \mathrm{GeV}$ & $10 \mu \mathrm{A}$ & $2 \times 10^{-4}$ \\
Mainz linac & $300 \mathrm{MeV}$ & $15 \mu \mathrm{A}$ & $1.5 \times 10^{-4}$ \\
MIT-Bates & $250 \mathrm{MeV}$ & $60 \mu \mathrm{A}$ & $10^{-2}$ \\
JLab & $1-11 \mathrm{GeV}$ & $100 \mu \mathrm{A}$ & $\mathrm{CW}$ \\
Mainz MAMI & $0.3-1.6 \mathrm{GeV}$ & $30 \mu \mathrm{A}$ & $\mathrm{CW}$ \\
Mainz MESA & $140 \mathrm{MeV}$ & $150 \mu \mathrm{A}$ & $\mathrm{CW}$ \\
\hline
\end{tabular}

energies are required for the backward angle measurements.

\subsection{Polarized beam}

A polarized beam is produced by shining circularly polarized laser light on a semiconductor crystal. Photoelectrons are accelerated in a static electric field and injected into the accelerator. The helicity of the laser light can be reversed quickly by using an electro-optical device called a Pockels cell. Over the years, the available polarization and intensity have advanced considerably $[65,66]$. Beams with polarizations of over $90 \%$ and intensities of more than $100 \mu A$ are now routinely available.

The period during which the helicity of the beam is fixed is called a window. Some experiments use pairs of "windows" with opposite helicity but randomly switch the order of the helicity to reject noise with a constant frequency. Other experiments use quartets with a pattern such as +--+ instead of pairs. The duration of the window can be 1-33 ms. The pattern is synchronized with the frequency of the power line to reject the potentially largest source of noise.

The asymmetry is calculated for each window pair or quartet. The statistical noise in PVES is usually determined by the observed width of the distribution of asymmetries, which may be as small as 200 ppm per window or quartet. For some experiments, this asymmetry measurement may be repeated as many as $4 \times 10^{9}$ times, reducing the statistical error to as small as $3 \mathrm{ppb}$ for the entire experiment. As an example, Fig. 6 shows the distribution of 25 million measurements of $A_{P V}$ from the HAPPEX-II [20] result with a width of $540 \mathrm{ppm}$.

\subsection{Helicity-correlated changes in the beam}

The position of an electron beam can be measured with a precision on the order of a micron during each beam window. A pair of such monitors can determine the position and angle of the beam on the target. The energy of the beam can be determined either by a position monitor at a position of high dispersion in the beam line or by timing in a beam line where the length of the path depends on the momentum of the beam. The monitors are used both to tune the source and accelerator to minimize the helicity-correlated beam differences and to correct the average asymmetries for any remaining systematic differences in the beam parameters [67].

The fluctuations of the beam parameters from window to window are usually large compared to the monitor resolution and may be large enough to increase the width of the asymmetry distribution, as in the one shown in 


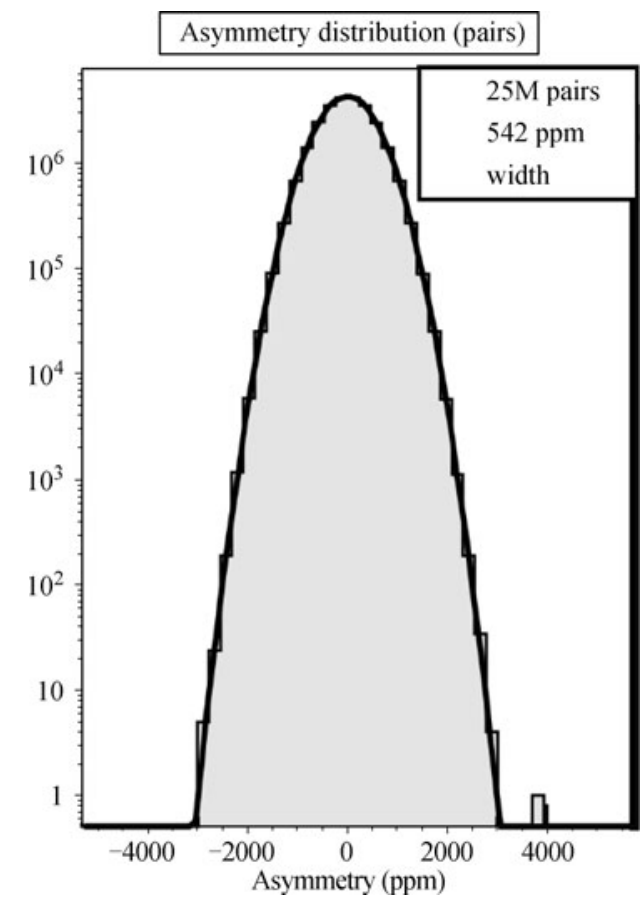

Fig. 6 Distribution of asymmetry measurements from the HAPPEX-II hydrogen runs.

Fig. 6. However, the monitor data may be used to correct the asymmetries so that the width is dominated by the statistics of the detected particles.

The dependence of the signal on the beam parameters can be calibrated by modulating the beam parameters during the run. An alternative is to use a regression analysis to remove correlations between the signal and the beam parameters. Regression analysis is useful if the resolution of the measurements of the beam parameters is better than the random noise in the beam and if the beam noise spans the beam parameter space. Because the modulation technique can be tuned to span the phase space, it is generally the more reliable technique.

The systematic difference in the beam properties between helicity states is most often created at the polarized source. Here, the handedness of the circular polarization of the laser beam determines the helicity of the electron beam. The Pockels cell creates this circular polarization, with positive and negative voltage settings selected to provide $\mathrm{a} \pm \frac{\lambda}{4}$ birefringence of linearly polarized light. The most obvious difference in the beam arises when the birefringence is imperfect, such that there is a residual component of linear polarization in the laser beam at the cathode that differs between the two polarization states. If this linear polarization is oriented along a preferred axis of the photocathode quantum efficiency, the beam helicity states will differ in intensity. This effect is typically used for feedback on the helicity-correlated beam intensity asymmetry, where small (typically $10^{-2}$ ) changes in the voltages are applied to the Pockels cell to keep the average beam intensity asymmetry small.

Although this effect can be easily managed by making small changes to the applied voltage, note that any gradient in the birefringence of any element (including the Pockels cell or the vacuum window) will create a position-dependent asymmetry. A linear gradient across the beam spot will evidently produce a helicitycorrelated difference in the beam centroid, whereas nonzero higher moments will result in helicity-correlated size or shape changes in the beam spot. These position effects can also be created by steering or lensing in the Pockels cell, as the most commonly used type $\left(\mathrm{KD}^{*} \mathrm{P}\right)$ is piezo-electric. These effects are controlled [68] by careful configuration of the optical components to avoid introducing gradients, by orienting the gradients to minimize the sensitivity, or, when these options are exhausted, by orienting effects such that they cancel each other as much as possible.

\subsection{Slow helicity reversals}

A common and very valuable technique for PVES measurements is to reverse the helicity of the beam in a manner unlike the fast reversal provided by the Pockels cell. The most common technique involves inserting, or rotating by $45^{\circ}$, a half-wave plate (HWP) upstream of the Pockels cell in the polarized source. This changes the orientation of the linearly polarized laser light by $90^{\circ}$, which in turn reverses the handedness of circular polarized light with respect to the Pockels cell voltage. This HWP reversal is minimally invasive; however, if it is the only change made, the beam helicity will change relative to the measurement synchronization, and the measured asymmetry will change sign.

There are two advantages to this technique. The first is that it can be used to test the measurement. The magnitude of the measured asymmetry should remain the same under the reversal. Verifying the same magnitude with opposite sign is therefore a test that the experiment is not subject to a false asymmetry that is determined by the Pockels cell voltage. Examples of such an asymmetry would be a pedestal shift in the integrating detector readout caused by the Pockels cell voltage or an uncorrected effect caused by piezo-electric steering in the Pockels cell. The second advantage is clear from the first: if there is an effect that changes sign with the Pockels cell, then when the measured asymmetry is corrected to reflect the beam helicity, the effect should be equal but opposite between the two HWP states. In this way, a slow reversal can be used to both demonstrate the existence of a false asymmetry and to cancel it out. The asymmetries of the 


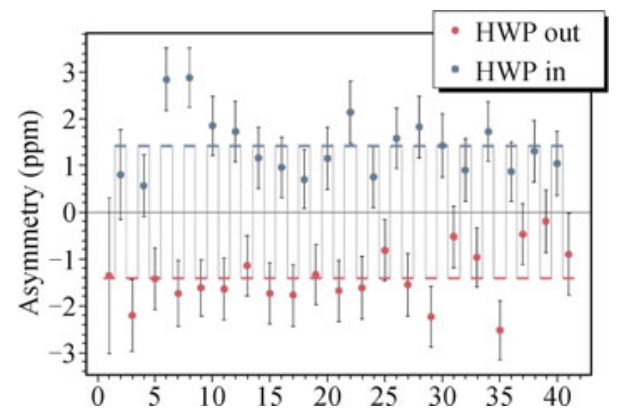

Fig. 7 The distribution of asymmetries in the HAPPEX-II hydrogen measurement, each averaged over about 12 hours, separated by the Half Wave Plate helicity reversal.

HAPPEX-II measurement, separated by HWP reversal, are shown in Fig. 7.

The HWP slow reversal is known to cancel helicitycorrelated beam differences arising from some, but not all, causes in the polarized source. It is typically an essential tool for reducing the beam differences to an acceptable level for an experiment. Other slow reversals are possible. Recent experiments at JLab [29, 69] have used spin manipulation in the low-energy injector with a combination of Wien rotators and solenoids to reverse the beam helicity with minimal changes to the beam properties. The E158 experiment [27] used a $6 \%$ change in beam energy, which changed the net spin precision in the arc entering the experimental hall and thus reversed the beam helicity.

\subsection{Targets}

PVES requires thick targets that can tolerate intense beams of $50-180 \mu \mathrm{A}$ in order to achieve the necessary statistics. The power deposited in the target is given by

$$
P=I L \rho \frac{\mathrm{d} E}{\mathrm{~d} X},
$$

where $P$ is the power (W), $I$ is the current (A), $L$ is the target length $(\mathrm{cm}), \rho$ is the density $\left(\mathrm{g} / \mathrm{cm}^{3}\right)$, and $\mathrm{d} E / \mathrm{d} X$ is the density of the energy deposited by an electron $\left[\mathrm{eV} /\left(\mathrm{g} / \mathrm{cm}^{3}\right)\right]$. This corresponds, for example, to 340 $\mathrm{W}$ for a typical 20 -cm-long liquid hydrogen $\left(\mathrm{LH}_{2}\right)$ target at $20 \mu \mathrm{A}$.

The most common target is $\mathrm{LH}_{2}$. These targets operate at a temperature near $20 \mathrm{~K}$ and a pressure of up to $220 \mathrm{kPa}$, with a density $\rho$ of $71 \mathrm{~kg} / \mathrm{m}^{3}$. High-power $\mathrm{LH}_{2}$ targets with $P<1 \mathrm{~kW}$ were developed at SLAC in the late 1960s, and advances were made at Caltech, including the SAMPLE [70], G0, and E158 targets. In recent years, JLab has taken the lead in target development.

The heat has to be removed from the target to maintain the low temperature and high density. A more subtle problem is that the beam heat in the target can cause boiling, which introduces rapid density fluctuations that increase the statistical error. Rapidly reversing the helicity of the beam addresses this problem by both reducing the size of the density fluctuations within a helicity window and also increasing the statistical error in each shorter window. Rastering the beam to a size of a few millimeters also reduces the density fluctuations. To maintain the desired properties of the $\mathrm{LH}_{2}$, the targets consist of a loop with the target cell that the beam passes through, a pump to maintain a high flow rate, a heat exchanger to cool the liquid, and a heater to balance the system if the beam trips off.

The highest-power target built to date was used by the Qweak [28] experiment. It was $35 \mathrm{~cm}$ long, absorbing 2.5 $\mathrm{kW}$ in a $180 \mu \mathrm{A}$ beam. The flow rate was on the order of 1 $\mathrm{kg} / \mathrm{s}$. The density fluctuations contributed only about 50 ppm at a flipping rate of $480 \mathrm{~Hz}$. Recent developments in computational fluid dynamics were critical for the design of the target.

Targets for planned experiments are even more ambitious. The JLab Møller target will be $150 \mathrm{~cm}$ long and absorb $5 \mathrm{~kW}$ at $85 \mu \mathrm{A}$, and the 60 -cm-long $\mathrm{P} 2$ target at Mainz will absorb $4 \mathrm{~kW}$ at $150 \mu \mathrm{A}$.

Other nuclear targets, such as carbon, can easily handle high beam currents. A more problematic target that is important for PVES is $\mathrm{Pb}$, which has a low melting point and poor thermal conductivity. For the PREx experiment, a diamond foil backing was used with a $\mathrm{Pb}$ target to conduct heat away from the beam spot to the foil. When the edges were cooled to $\sim 20 \mathrm{~K}$, it was possible to operate the target at $70 \mu \mathrm{A}$ without melting.

\subsection{Spectrometers}

The task of the spectrometer in a PVES experiment is to identify the desired, usually elastic, events, and also eliminate as much background as possible. Magnets are ideal for this purpose; in a magnetic field, the deflection of a scattered electron depends on its momentum. Neutral particles such as photons, which travel in straight lines, can be blocked by collimators. The position of an electron in the detector region can be quite sensitive to the momentum, facilitating the rejection of inelastic scatters, even if the energy lost is relatively small. There are a number of possible configurations for magnets, many of which have been used for PVES. Table 4 lists the spectrometers used in past and future PVES experiments.

In this section, we will discuss the general properties of spectrometers. In the following subsections, we discuss selected spectrometers used in PVES programs in detail.

Quadrupole magnets create a magnetic field gradient 
Table 4 Apparatus for selected parity experiments. For magnets, $\mathrm{Q}=$ quadrupole, $\mathrm{D}=$ dipole.

\begin{tabular}{ccccc}
\hline Experiment & Magnets & Detector & Count & $e^{-}$angles $\left(^{\circ}\right)$ \\
\hline SLAC E122 & DQD & Pb Glass & No & 4 \\
Mainz & None & Air C & No & 130 \\
MIT-Bates & Q & Lucite C & No & 35 \\
SAMPLE & None & Air C & No & 146 \\
HAPPEX-I & QQDQ & Pb-Lucite & No & 15 \\
G0 & Toroid & Scintillator & Yes & $6-20 ; 110$ \\
A4 & None & PbF $_{2}$ & Yes & $35 ; 145$ \\
SLAC E158 & QQQQ & Cu-Quartz & No & 2 \\
HAPPEX-II & DQQDQ & Cu-Quartz & No & 5 \\
PREx -I & DQQDQ & Quartz & No & 5 \\
HAPPEX-III & QQDQ & Pb-Lucite & No & 15 \\
PVDIS & QQDQ & Pb Glass & Yes & 19 \\
PREx -II & DQQDQ & Quartz & No & 5 \\
CREx & DQQDQ & Quartz & No & 4 \\
Qweak & Toroid & Pb-Quartz & No & 5 \\
Møller & Toroid & Quartz & No & $0.3-1$ \\
SoLID & Solenoid & Package & Yes & $22-35$ \\
P2 & Solenoid & Quartz & No & 20 \\
Mainz C & Solenoid & Quartz & No & 40 \\
\hline & & & &
\end{tabular}

that serves to focus charged particles, where the focal length depends on the momentum. Quadrupoles are typically used in conjunction with other magnets, but some spectrometers use only quadrupoles. The ${ }^{12} \mathrm{C}$ experiment at Bates used a pair of quadrupoles. The SLAC Møller experiment used a series of four quadrupoles as the spectrometer.

The simplest magnet is the dipole, in which a relatively uniform field is created in a gap in an iron yoke. The iron yoke minimizes the current required to create the field and allows flexibility in the placement of the coils driving the field. The trajectories of the scattered electrons are usually perpendicular to the field lines, creating the most efficient bending. Dipoles are vital for spectrometers with the highest-energy particles or the most precise resolution. The performance of dipoles is usually enhanced by using quadrupoles. The SLAC E122 experiment used a spectrometer with two dipoles and a quadrupole in the middle. The high-resolution spectrometers (HRSs) used for the HAPPEX experiments used a spectrometer with three quadrupoles and one dipole.

Spectrometers with quadrupoles and dipoles tend to have a small angular acceptance. For scattering at small angles ( $15^{\circ}$ or less), this is acceptable, as the total solid angle available at forward angles is limited. For PVES experiments operating at larger angles, different types of magnets are required. Two choices are the toroid and the solenoid. For a toroid, the field lines are in the $\hat{\phi}$ direction, so the field is again perpendicular to the trajectories. The coils for the toroid must be in the acceptance, so the $\Delta \phi$ acceptance is limited, typically to $50 \%$. The G0, Qweak, and proposed JLab MOLLER experiments all use toroids. Toroidal magnets are usually designed and built especially for the experiments in which they are used.

Solenoid magnets have the advantage of a full azimuthal acceptance. At small angles, however, the trajectories are roughly parallel to the magnetic field lines, so the deflection is limited, and good resolution is harder to achieve. However, with a large solenoid, thin targets, and relatively low-energy beams, a solenoidal spectrometer can have impressive performance in terms of both the acceptance and energy resolution [71]. Typical trajectories for such a system are shown in Fig. 8. Future experiments in $\mathrm{Pb}$ or $\mathrm{C}$ might use this method.

The P2 experiment at Mainz [30] will use a solenoid but with a thick $\mathrm{LH}_{2}$ target to measure the weak charge of the proton. Because the beam energy is low (150 $\mathrm{MeV}$ ), and the inelastic events must create a $135 \mathrm{MeV}$ $\pi^{0}$, crude resolution is ample. A set of trajectories is shown in Fig. 9.

A solenoid is also planned for the SoLID spectrometer for a measurement of PVDIS. In contrast to the case for the P2 spectrometer, the scattered electrons have an energy between 2 and $5 \mathrm{GeV}$, and the bend angles are small. Therefore, tracking detectors are required to measure the momentum.

To achieve the largest possible solid angle acceptance, some PVES experiments avoid magnets altogether. At low energies, electrons can be isolated from background by using a gas Čerenkov detector [16] or, at somewhat higher energies, a calorimeter, as was done for the A4 program at Mainz.

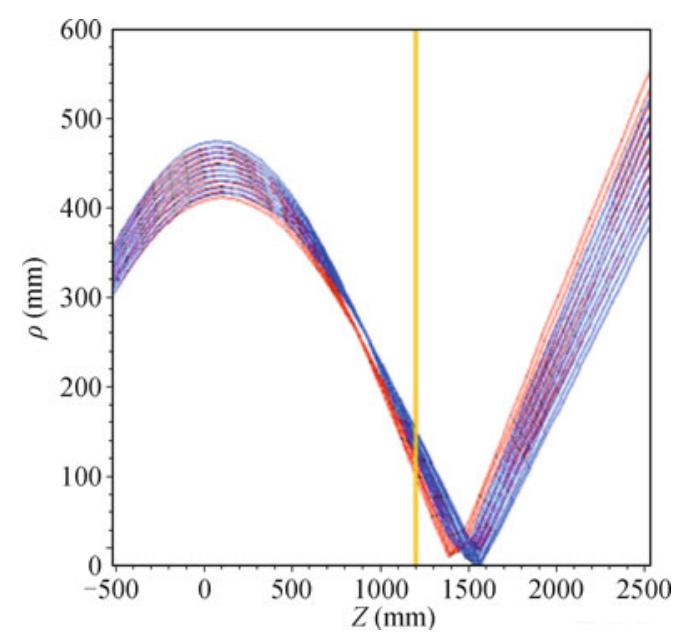

Fig. 8 Trajectories for elasitic and inelastic electrons scattered from a thin target in a $1200 \mathrm{~mm}$-long solenoid. At $1000 \mathrm{~mm}$, the elastic and inelastic events are separated, and the inelastic events can be blocked by a collimator. 


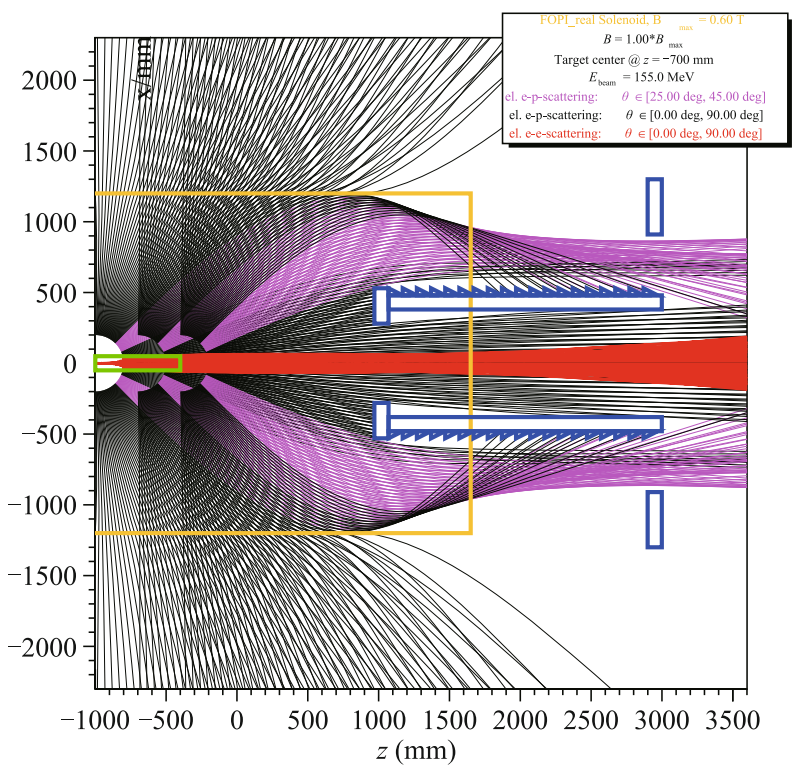

Fig. 9 Raytraces for the P2 spectrometer. Elastic events in the acceptance region are in magenta. Low energy Møller electrons, shown in red, are confined to a region near the beam due to the strong magnetic field. The detectors are located in a region of low background at $3000 \mathrm{~mm}$.

\subsubsection{JLab high-resolution spectrometer}

Each of the pair of HRSs in Hall A at JLab comprises two quadrupoles, a dipole with a bend angle of $45^{\circ}$, a quadrupole, and a "standard detector package" [72]. The path length of the detected particles is $24 \mathrm{~m}$. The spectrometers can detect particles with momenta up to 3.2 $\mathrm{GeV} / \mathrm{c}$. Each has a solid angle acceptance of $6 \mathrm{msr}: \pm 60$ mrad in the vertical direction and $\pm 28 \mathrm{mrad}$ in the horizontal direction. The momentum acceptance is $\pm 4 \%$. The spectrometers could view a target $10 \mathrm{~cm}$ long at $90^{\circ}$; they used a $15 \mathrm{~cm}$ target at $12.5^{\circ}$ and a $20 \mathrm{~cm}$ target at $6^{\circ}$.

The HRS is designed to detect reactions in which a single proton was ejected with sufficient resolution to determine the energy level of the orbital of the proton. This requires an energy resolution of $2 \times 10^{-4}$ (FWHM), corresponding to an energy resolution at a level of a few hundreds of kilo-electron volts. The PVES program was not considered in the design. However, the HRS has proved to be ideal for PVES [19]. It was used for six different completed PVES experiments, and two experiments plan to use it in the future.

The PVES experiments generally use the HRS positioned at the most forward angle of $12.5^{\circ}$, where the acceptance is between $10.9^{\circ}$ and $14.1^{\circ}$. The solid angle of the HRS, $6 \mathrm{msr}$, is $15 \%$ of the total available solid angle between those angles, or about $30 \%$ of what could be obtained with a toroidal spectrometer. This loss of acceptance is more than made up for by the excellent energy resolution and background rejection. The FOM for elastic scattering from the proton is maximized at the largest energy and smallest angle, corresponding to $Q^{2} \sim 0.5(\mathrm{GeV} / \mathrm{c})^{2}$. The main disadvantage of the HRS is the lack of acceptance at large angles.

The HRS can also be operated with the addition of a pair of septum magnets that allow the scattering angle to be reduced to $6^{\circ}$. This allows experiments to run at much smaller $Q^{2}$ values of about $0.1(\mathrm{GeV} / \mathrm{c})^{2}$.

The standard detector package consists of drift chambers for tracking, a Čerenkov counter for particle identification, and a $\mathrm{Pb}$ glass electron calorimeter. The full resolution requires tracking, which was not designed to operate at the high rates of PVES. However, using only the position of the events at the focal plane yields a resolution of better than $0.5 \%$. For example, in the ${ }^{4} \mathrm{He}$ phase of the HAPPEX experiment, events that lost more than $15 \mathrm{MeV}$ missed the detector. This resolution was ample, as the first excited state in ${ }^{4} \mathrm{He}$ occurs at $20 \mathrm{MeV}$. Thus, a single detector could identify the elastic events. The large dispersion of the HRS, $12.5 \mathrm{~m}$, is the key to the hardware resolution.

The standard HRS detector packages were designed for high-resolution tracking at rates that were low relative to those required for parity-violation measurements. The tracking detectors were used, with very low beam currents to reach sufficiently low rates, for the following studies:

1) Calibration of the optics and central scattering angle;

2) Measurement of the average $Q^{2}$ of the accepted distribution;

3) Measurement of the response of the detector;

4) Measurement of the size and distribution of the backgrounds.

These studies were critical to obtaining small systematic errors.

The HRS was also used to measure PVDIS [73, 74], but with a different detector and readout configuration. First, to reject pions, a coincidence between the lead glass and gas Čerenkov counters was used. Second, a special DAQ system was used to handle the high rates.

\subsubsection{G0 toroidal spectrometer}

The G0 experiment [75] at JLab was designed to measure $A_{P V}$ for elastic scattering from hydrogen over a wide range of angles and thus a large range of $Q^{2}$. The heart of the G0 experiment was a large, superconducting toroidal spectrometer. The eight acceptance regions, called oc- 
tants, were between the eight coils comprising the toroid. The configuration allowed a total $\Delta \phi$ acceptance of $41 \%$ of the full azimuth. The experiment was conducted in two phases. The first phase measured $A_{P V}$ for electrons scattered in the forward direction at a beam energy of $3.03 \mathrm{GeV}$, and the second made measurements at backward angles with beam energies of 0.359 and $0.684 \mathrm{GeV}$.

In contrast to most PVES experiments, which detect the scattered electron, the first phase of G0 detected the recoil proton in the range $52^{\circ}<\theta_{p}<77^{\circ}$, corresponding to electrons scattered between $6^{\circ}$ and $21^{\circ}$. The electrons were not detected. The protons for each octant were detected by a set of 15 scintillators, with a total of 120 detectors in all. In typical operation, the continuous-wave (CW) JLab accelerator delivers beam bunches to each experimental hall with a spacing of 2 ns. For the G0 experiment, the source laser was pulsed at a lower frequency, so that only one in 16 cycles was generated at the source, and the beam bunches were separated by $32 \mathrm{~ns}$. Consequently, the time of flight could be used to separate the slower protons from pions and other backgrounds.

Using $700 \mathrm{~h}$ of data in the forward-angle configuration, the G0 collaboration published asymmetries for 18 bins in $Q^{2}$ covering the range $0.122-0.997(\mathrm{GeV} / \mathrm{c})^{2}$. For the lower $Q^{2}$ points, the bins corresponded to individual scintillators. The three highest $Q^{2}$ bins came from one scintillator and were separated using time-of-flight data. Over that range, the asymmetry varied by almost 2 orders of magnitude, from about 1 to $40 \mathrm{ppm}$. The asymmetries included some of the smallest measured in parity-violating electron-proton scattering to that date.

Even though some of the asymmetries were small and precisely measured, the uncertainties due to helicitycorrelated beam changes (see Section 3.4) were at the impressively small level of $0.01 \mathrm{ppm}$ and entirely negligible. However, there were a number of more important systematic errors that were unique to the G0 apparatus. At the high-rate and low-asymmetry points, the rates in the scintillators were on the order of $2 \mathrm{MHz}$, resulting in $10 \%-15 \%$ dead time corrections that produced uncertainties on the order of $0.05 \mathrm{ppm}$. About $0.1 \%$ of the beam was in the nominally unoccupied beam bunches. These out-of-time electrons were detected in the beam intensity monitor, but the corresponding out-of-time signal protons were not included in the time-of-flight cut. The correction for this effect was surprisingly large, 0.71 $\pm 0.14 \mathrm{ppm}$. For the higher- $Q^{2}$ points, the uncertainties in the backgrounds were more important. One problem was that the very small backgrounds from decays of polarized hyperons produced in the target had very large asymmetries due to the large parity violation in their decays.
For the second phase of the experiment, the setup was significantly reconfigured to measure $A_{P V}$ at backward scattering angles. The spectrometer was turned around and configured so that backward-scattered electrons would be detected on the scintillator bars. An additional set of scintillators located near the target were used in coincidence to define the kinematic bins. An aerogel Čerenkov detector was used in anticoincidence to reject pions.

In contrast to the situation for small angles, where $A_{P V}$ varies rapidly with the kinematics, the asymmetry is fairly flat at backward angles, and only one $Q^{2}$ point was obtained at each of the two beam energies studied. Both hydrogen and deuterium targets were used to help reduce the uncertainties from radiative corrections (see Section 2.4). Two $Q^{2}$ points were measured, 0.221 and $0.628(\mathrm{GeV} / \mathrm{c})^{2}$. For the low- $Q^{2}$ point at back angles, the dominant term in the asymmetry (the $A_{M}$ term; see Section 2.4) is not suppressed by $1-4 \sin ^{2} \theta_{W}$, so the asymmetries are much larger than for the forward data. One of the most important systematic uncertainties arose from dead time corrections, especially for the deuterium data, where the background rates from pions were the highest.

\subsubsection{Crystal spectrometer at Mainz}

At Mainz, a spectrometer that was developed for PVES achieved a large solid angle by dispensing entirely with a magnet. The idea was to use an array of $1022 \mathrm{PbF}_{2}$ crystals $[76,77]$ at a distance of $0.5 \mathrm{~m}$ from the target to accept events scattered from $30^{\circ}$ to $40^{\circ}$, achieving a total solid angle of $0.6 \mathrm{sr}$. The crystals rely only on Čerenkov light, so they are very fast and are insensitive to slow background particles. Given the CW beam and the large number of channels, the event rate of $5 \times 10^{7} / \mathrm{s}$ was manageable. An elaborate system of electronics sums all combinations of nine adjacent crystals and produces histograms of the energy spectra. Because the beam energy is 854 or $570 \mathrm{MeV}$, the $3.9 \% / \sqrt{E}$ resolution is sufficient to reject inelastic events, which must be more than 100 $\mathrm{MeV}$ below the elastic peak. The dominant background was due to quasi-elastic scattering from the aluminum target windows. There was also less than $1 \%$ background due to photons from $\pi^{0}$ decay.

The apparatus could also be rotated $180^{\circ}$ about a vertical axis through the target to subtend back angles between $140^{\circ}$ and $150^{\circ}$. In this configuration, a layer of plastic scintillator was added in front of the $\mathrm{PbF}_{2}$ crystals, allowing the separation of electrons from the photons from $\pi^{0}$ decay. The asymmetry of the remaining photon background was determined by measuring the 
asymmetry of the non-coincidence events between the plastic and crystal scintillators. Conversion of photons into $e^{+} e^{-}$pairs formed a significant background, which was corrected for using a Monte Carlo simulation. Measurements of $A_{P V}$ from the proton were made using this apparatus in the forward angle at $Q^{2}$ points of 0.108 and $0.23(\mathrm{GeV} / \mathrm{c})^{2}$ and in the backward angle at $Q^{2}=0.22$ $(\mathrm{GeV} / \mathrm{c})^{2}[24-26]$.

\subsubsection{The Qweak spectrometer}

The Qweak spectrometer was designed to measure $A_{P V}$ for hydrogen at a sufficiently low $Q^{2}$ that $Q_{W}(p)$ could be measured. The heart of the Qweak spectrometer was an eight-coil toroid about $3 \mathrm{~m}$ long. In contrast to those of the G0 toroid, the coils for Qweak were resistive. The toroid had a maximum field of $0.5 \mathrm{~T}$ and an $\int \boldsymbol{B} \cdot \mathrm{d} \boldsymbol{l}$ of $0.89 \mathrm{~T}-\mathrm{m}$. It focused electrons from a $\mathrm{LH}_{2}$ target $6.5 \mathrm{~m}$ upstream onto quartz bars $5.7 \mathrm{~m}$ downstream. The electrons were bent away from the beam. Inelastic events had larger bending angles and missed the detectors. The acceptance was defined by a set of three collimators, which gave a scattering angle of $7.9^{\circ} \pm 3^{\circ}$ and an azimuthal acceptance of $49 \%$ of $2 \pi$.

The detectors were $100 \mathrm{~cm}$ long by $18 \mathrm{~cm}$ wide by 1.25 $\mathrm{cm}$ thick and oriented in an octagon. Each detector had an event rate of $640 \mathrm{MHz}$. The detectors were located in a heavily shielded cave to minimize backgrounds. A set of drift chambers could be inserted into the spectrometer for special data runs with very low intensity to study the detector response, spectrometer acceptance, and backgrounds. Results of those studies were used to benchmark the simulation that was used to determine the $Q^{2}$ value of the $A_{P V}$ measurement.

\subsubsection{JLab SoLID spectrometer}

The SoLID collaboration has designed a solenoidal spectrometer to implement three physics programs:

1) PVDIS with deuterium, proton, and nuclear targets [78];

2) Semi-inclusive DIS (SIDIS) with polarized targets, especially ${ }^{3} \mathrm{He}$, where a pion is also detected in the final state;

3) Measurement of the cross section for the production of the $J / \Psi$ particle near threshold.

This proposed facility is unique at JLab in that it can operate with both large acceptance and high luminosity.

The SoLID spectrometer uses a $1.5 \mathrm{~T}$ superconducting solenoid $3.5 \mathrm{~m}$ long and $2.9 \mathrm{~m}$ in diameter. The magnet was formerly used in the CLEO facility at Cornell.
In the PVDIS configuration, the magnet is instrumented as follows. A hydrogen or deuterium target is placed at the center of the magnet. A set of gas electron multiplier (GEM) chambers provides tracking of the scattered particles in the high-rate environment. Electrons are identified by a gas Čerenkov detector and a shashlik-type electromagnetic calorimeter. A set of baffles, which block the photons from the target as well a number of other backgrounds, divides the solenoid into 30 independent sectors. The baffles reduce the acceptance by about $70 \%$ but allow the experiment to run with a beam current of $50 \mu \mathrm{A}$ on the target. Figure 10 shows a schematic view of the SoLID spectrometer.

Because the values of $Q^{2}$ are quite large, up to 8 $(\mathrm{GeV} / \mathrm{c})^{2}$, the asymmetries are large by comparison, so the counting rates can be low. Thus, traditional coincidence techniques are used to identify the events. The kinematics for each event is determined individually, so many different kinematic bins can be obtained simultaneously.

In the SIDIS and $J / \Psi$ configuration, the target is located upstream of the magnet. An additional Čerenkov counter is installed to separate pions from kaons. A multi-gap resistive plate chamber detector is also used for particle identification using time-of-flight data.

\subsection{Møller spectrometers}

In Møller scattering, $A_{P V}$ is largest for $y=1 / 2$. When an integrating spectrometer is used, an acceptance in the range $1 / 4<y<3 / 4$ is optimal. Because the beam and target particles are identical, a spectrometer can detect each scatter twice, one electron characterized by $y_{1}$ and the other $y_{2}=1-y_{1}$. Therefore, it is sufficient to accept, for example, $1 / 4<y \leqslant 1 / 2$. The scattered electrons are very forward, as is evident from Eq. (13), so Møller

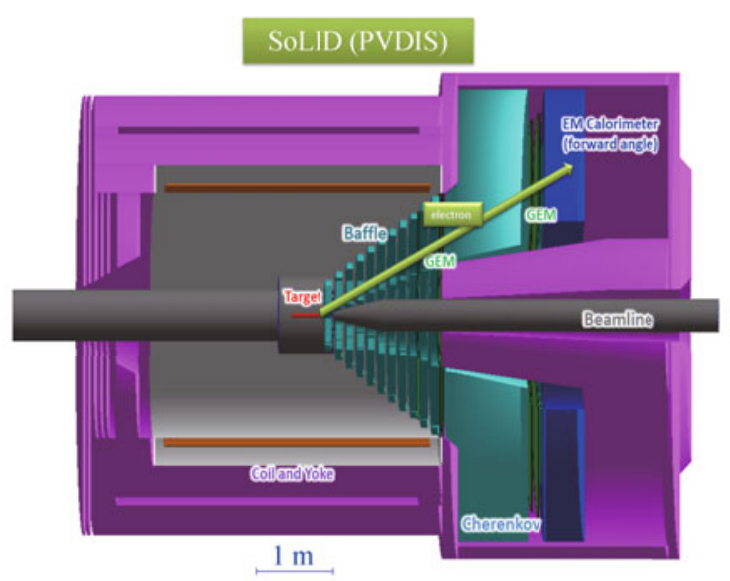

Fig. 10 Diagram of the SoLID spectrometer in the PVDIS configuration. 
experiments require specialized spectrometers.

At $50 \mathrm{GeV}$, the energy of the SLAC E158 experiment, $1 / 4<y \leqslant 1 / 2$ corresponds to laboratory scattering angles from 4.5 to $8 \mathrm{mrad}$. The spectrometer design was based on a chicane and four quadrupole magnets. The chicane passes electrons in the desired energy range but blocks most of the background. The quadrupoles focus the Møller electrons onto a circle in which the detector is placed. Ring-shaped collimators around the beamline are used to further reduce the background and define the angular acceptance. The supports for these collimators limit the azimuthal acceptance to about $80 \%$ of $2 \pi$.

For the new JLab $12 \mathrm{GeV}$ experiment, a design based on toroidal magnets is being developed. A drawing of the apparatus is shown in Fig. 11. The toroids have seven open sectors accepting $50 \%$ of $\Delta \phi$. The energy accep-

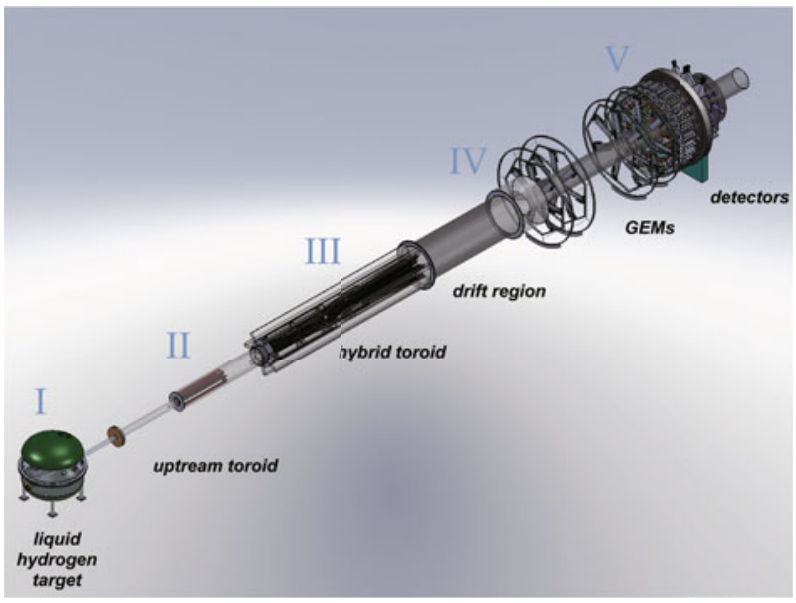

Fig. 11 Drawing of the MOLLER spectrometer. Refgon I is the target, region II is the first toroid, region III is the main toroid, region IV is the drift retion with removable GEM detectors for the calibration of the $Q^{2}$, and region $\mathrm{V}$ contains the integrating detector package. III is in the PVDIS configuration.

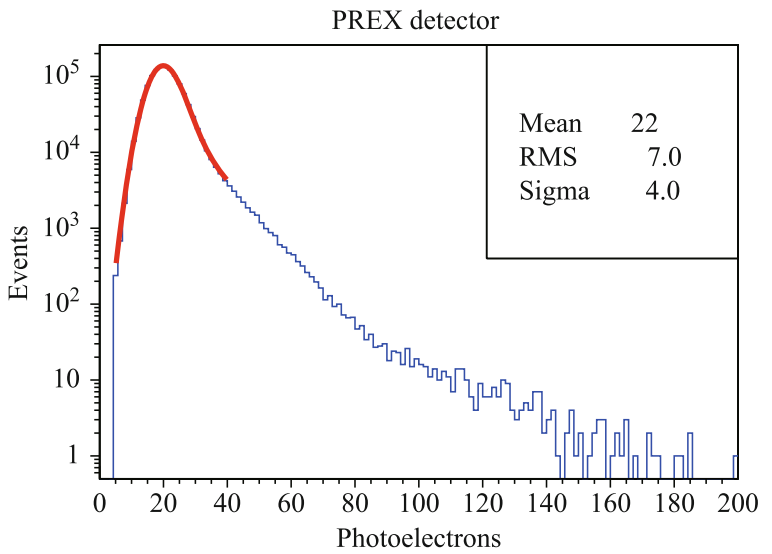

Fig. 12 Pulse height spectrum from the thin quartz detector used for the PREx experiment. The Sigma comes from the Gaussian part of the distribution. The RMS is almost twice as large and is about a third of the average signal, resulting is a $10 \%$ loss in statistics. tance is in the range $1 / 4<y<3 / 4$, corresponding to lab angles of 6-18 mrad. However, because Møller scattering involves identical particles, an acceptance of nearly $100 \%$ is achieved in the desired $y$ range.

\subsection{Detectors}

A wide variety of detectors have been used for PVES spectrometers. One reason is that the detector must match the spectrometer, and a variety of different spectrometers have been used. There are two classes of detectors for PVES experiments; one integrates the signal over a beam window, and the other counts individual events that are summed over a beam window. For integrating detectors, often only a few channels of electronics are used. For counting detectors, usually many detectors must be employed to keep the rates below about $10^{6}$ per channel in order to minimize systematic errors due to pileup and dead time effects. Counting particles can also take advantage of coincidences between various detectors, which is a powerful way to reject both detector noise and background events.

\subsubsection{Integrating detectors}

One of the groundbreaking aspects of the Prescott experiment is that it was based on integration of the signal. In this method, the current from a detector is integrated over the duration of a helicity window, and the total charge is read out and used to compute the asymmetry. The main reason integration was needed was that, for the pulsed accelerator with a duty factor of $\sim 10^{-4}$, the observed event rate was much too high to count. A major bonus of the technique is that it requires no accidental or dead time corrections.

Integrating detectors for PVES are usually Čerenkov detectors, which are insensitive to nonrelativistic background particles. One potential disadvantage of integrating detectors is that there is some loss of statistics due to the root mean square (RMS) resolution $\sigma$ of the detector; the statistical error for $N$ counts is given by

$$
\frac{\Delta A_{P V}}{A_{P V}}=\frac{1}{\sqrt{N}} \sqrt{1+\frac{\sigma^{2}}{\langle S\rangle^{2}}},
$$

where $\langle S\rangle$ is the average signal size. A fractional energy resolution of $\sigma /\langle S\rangle \sim 15 \%$ is ample, and even 30\% resolution costs only $10 \%$ of the running time.

Lead glass calorimeters, with a resolution on the order of $5 \% / \sqrt{E}$, where $E$ is the energy of the detected electrons in giga-electron volts, are ideal and were used for the SLAC E122 experiment. All of the energy of the electrons produces signal, whereas slow particles produce no 
Čerenkov light, and fast pions deposit little energy. Unfortunately, the rates for many PVES experiments are so high that the lead glass becomes blackened by radiation damage and no longer provides signal.

A common alternative is a detector made of layers of a high- $Z$ radiator such as $\mathrm{Cu}$ or $\mathrm{Pb}$ and a transparent material. There are two contributions to the width $\sigma$ of the response of such detectors: photon statistics and shower fluctuations. The photon statistics are maximized by having a large fraction of transparent material, and the shower fluctuations are minimized by using many layers. A practical detector requires a compromise between these two features.

A good example of an integrating device is the detector used for the HAPPEX-I experiment. It is $1.5 \mathrm{~m}$ long and $10 \mathrm{~cm}$ wide, and covers the region of the elastic peak in the focal plane of the spectrometer. It is made of a layer of 12.8-mm-thick $\mathrm{Pb}$ followed by five layers of 1.27-cm-thick acrylic each followed by a layer of 6.4 mm-thick $\mathrm{Pb}$. The resolution, $\sigma /\langle S\rangle$, is $15 \%$, which is acceptable for an integrating detector but much worse than that of lead glass. The resolution is limited by the shower fluctuations; there is ample photon statistics. The radiation dose was 40 Gy for the 30-day run, which was low enough to use the acrylic but much too high to use conventional $\mathrm{Pb}$ glass. The position dependence of the signal was $9 \% / \mathrm{m}$.

For the HAPPEX-II experiments, the rate was too high even for acrylic, so the detector was made of brass and quartz sheets. For E158, where the detected electrons had an energy of more than $12 \mathrm{GeV}$, a Cu calorimeter with quartz fibers was used. Quartz fibers are expensive, and the resolution was limited by the number of fibers in the detector and thus the total amount of light produced in the fibers.

For electrons with energies below 1 or $2 \mathrm{GeV}$, the shower fluctuations become more important. One solution is to use a thin piece of quartz with a thickness in the range of $0.5-1.0 \mathrm{~cm}$. If it is any thicker, high-energy tails, due primarily to delta rays, increase the resolution. As the detector becomes thinner, the photon statistics increases the width. An example of the response of a thin quartz detector is given in Fig. 12. The performance is inferior to that of the HAPPEX detector described above but is still acceptable. For the Qweak experiment, $2 \mathrm{~cm}$ of $\mathrm{Pb}$ was placed in front of a 1.25-cm-thick quartz bar to enhance the signal from the $1.1 \mathrm{GeV}$ electrons by a factor of 10 . The shower fluctuations were large with this arrangement, giving $\sigma /\langle S\rangle \sim 45 \%$. Thin quartz is the most common detector for the Generation 4 PVES experiments.

\subsubsection{Counting detectors}

With the development of $\mathrm{CW}$ electron accelerators, PVES experiments could be conducted using counting and coincidence techniques. The key was the use of detectors with many elements so that the rate in each individual elements was relatively low. The G0, A4, and JLab PVDIS experiments all used counting techniques.

\subsection{Electronics}

The high rates of PVES experiments are completely incompatible with the electronic and DAQ systems used for cross section measurements. Custom electronics were developed for each experiment.

For integrating experiments, the charge from the detectors is collected on a capacitor during each helicity window, and the voltage is read by an analog-to-digital convertor (ADC) at the end of the window. This procedure avoids the problem of trying to record the massive number of individual events. A very high-resolution ADC system with the smallest possible noise level is required. One advantage of integrating is that the noise is easy to measure from the width of the asymmetries between the window pairs, and the introduced error is accurately known. The noise appears as a statistical error, not a systematic one. An alternative integration technique uses a flash ADC, which converts at a high repetition rate, to sample the signal over the helicity window and return just the average of these samples.

For counting experiments, it is difficult to handle the massive amount of date produced. The typical method is to develop for each channel an elaborate electronic system that produces histograms. Only the histograms are recorded for further analysis, reducing the quantity of data to a manageable amount.

\subsection{Polarimetry}

The measured asymmetry is proportional to the polarization $P_{e}$ of the beam. For measurements with a small fractional error, $\delta A_{P V} / A_{P V}$, the error in $P_{e}$ can be significant, and attention must be paid to measuring $P_{e}$ during the experiment. $P_{e}$ is measured using either Møller scattering or Compton scattering.

\subsubsection{Møller polarimeters}

The first beam polarimeter at a high-energy accelerator was based on Møller scattering from a thin magnetized iron foil [79]. This process depends on a calculable nonparity-violating asymmetry in the scattering of polarized electrons from the polarized electrons in the target. 
There are several limitations to the precision that can be obtained using Møller scattering polarimeters.

1) The polarization measurement cannot be performed simultaneously with the main experiment, as the electron beam is degraded significantly by the magnetic foils used.

2) The beam current is limited by target heating to a few microamps, whereas the experiment is performed with a current more than ten times higher than this.

3) There may be significant uncertainties in the polarization of the electrons in the foil.

4) The polarized electrons in the foil correspond to a particular atomic shell; the other electrons are unpolarized. The scattering depends slightly on the shell of the electron struck in the iron. In particular, the polarized electrons are in the $\mathrm{D}$ shell. The inner electrons, which are unpolarized, have more momentum and broaden the distribution of the scattered electrons, a phenomenon called the Levchuk effect [80]. The corrections for this effect introduce an additional uncertainty.

The first Møller polarimeters used a high-permeable magnetic alloy that saturates at low magnetic fields. Additional uncertainties arise from the exact degree of saturation. An improved method uses a high polarizing field (greater than $3 \mathrm{~T}$ ) and a pure iron target. In this case, the magnetization of the foil may be driven to a repeatable maximum value ("saturation"); this saturation magnetization has been measured to high precision under ideal conditions in the laboratory. In this case, the precision can be reduced to better than $1 \%$.

A better Møller polarimeter can be made by using a cryogenic atomic hydrogen target in a high magnetic field [81]. Only one polarization state of the hydrogen atoms is trapped in the magnetic field, so the polarization of the electron is precisely known. The Levchuk effect is absent. For proposed high-precision experiments, especially with low-energy beams, this is the most promising method.

\subsubsection{Compton polarimeters}

Another method of measuring the beam polarization is to scatter the electron beam from polarized laser light. The polarization of the "target" laser can be determined much more precisely than that of an electron in an iron foil. One of the challenges of a laser polarimeter is that the photon "target" is not very thick, so the rates tend to be low, and backgrounds can be a problem.
In Compton scattering, either the scattered electron, the scattered photon, or both in coincidence can be used to measure the asymmetry. Each method has different systematic errors, so comparing them is an important check. The coincidence method has the advantage of reducing backgrounds.

The kinematics of Compton scattering varies immensely with the energy. The technique is easiest with higher-energy electrons. Here, the asymmetries are much larger, the energy of the ejected photon is larger, and the energy loss of the scattered electron is larger.

Compton polarimetry has been successful with a beam energy of only $1 \mathrm{GeV}$, where the scattered photon has an energy only on the order of tens of mega-electron volts. Signal integration was found to be a powerful technique for operating in this range [82]. No energy calibration of the individual photons is needed, and the average energy depends on the precisely known beam energy. Dead time and pileup effects are absent. Backgrounds can be subtracted by taking data with the laser off. The main trick is to achieve an accelerator tune at which the background rate is less than the signal rate, which can be achieved with some effort.

One of the more important systematic corrections for Compton polarimetry is the determination of the circular polarization of the photon beam. Although the laser polarization can be characterized well before the laser passes into the electron beam vacuum system and measured on the exit as well, it is more difficult to measure the polarization in the interaction region (accounting for the vacuum entry windows, for instance). When the photons are in the high-finesse cavity needed to obtain sufficient rates, the task is especially challenging, as the power level is low when the cavity is not on resonance owing to the highly reflective cavity input mirror. However, a technique has recently been developed that determines this parameter at the level of $0.2 \%$ [83].

\section{$3.12 Q^{2}$ calibration}

The asymmetry $A_{P V}$ depends on the $Q^{2}$ value of the events, and for experiments where the asymmetry is measured to high precision, measuring $Q^{2}$ to the required precision may be a challenge. $Q^{2}$ depends on the incident and scattered energies and scattering angle $\theta$. The energy of beams in electron accelerators is precisely known to better that $0.01 \%$, as this quantity is required for many of the unpolarized experiments in the laboratory. Thus, the main problem is to precisely measure the angle. In addition, because $Q^{2}$ usually varies extensively over the acceptance, the weighted average is required. For some experiments, this is accomplished by lowering the beam 
current to lower the count rate and using a tracking system. For many experiments, the tracking system can be surveyed with sufficient precision to calibrate the angle. In other experiments, tracking chambers are not used, and the accepted kinematics are deduced only through the surveyed geometry, with the average over the acceptance and detector response modeled in simulation.

For a high-resolution system such as the Hall A HRSs, a water target can be used to calibrate the scattering angle precisely and independently of a physics survey. This technique uses the fact that electrons elastically scattering from different nuclei $(\mathrm{H}, \mathrm{O}$, and $\mathrm{Fe}$ from the target window) have different recoil energies, which are reflected in the scattered energy:

$$
\frac{\delta E}{E}=\frac{\theta^{2}}{2} \frac{E}{M_{T}} .
$$

The differences in the energies of the electrons scattered from the various targets is easily resolved, as can be seen in the data in Fig. 13. The value of $\theta$ can be accurately inferred from the data.

\section{Results and implications}

\subsection{Strange form factors}

The charges in the nucleon come from the three valence quarks plus a sea of quark-antiquark pairs. Before 1990, it was assumed that the angular momentum of the proton came from the spin of the three valence quarks and that the sea was totally symmetric. As discussed in Section 1.1, measurements of spin structure functions [13] indicated that relatively little angular momentum came from the valence quarks, and the search was on for the other contributions. Sea quarks were high on the list. Then Kaplan and Manohar [14] asked whether, if the sea was that complicated, was it not reasonable that the strange

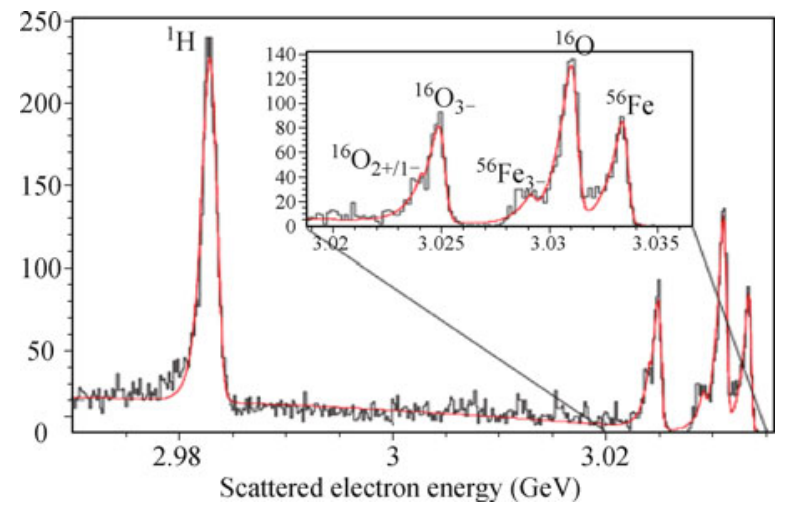

Fig. 13 Energy spectrum in the HRS for a water target. Peaks from different target masses are clearly distinguished and their separation can be precisely measured.

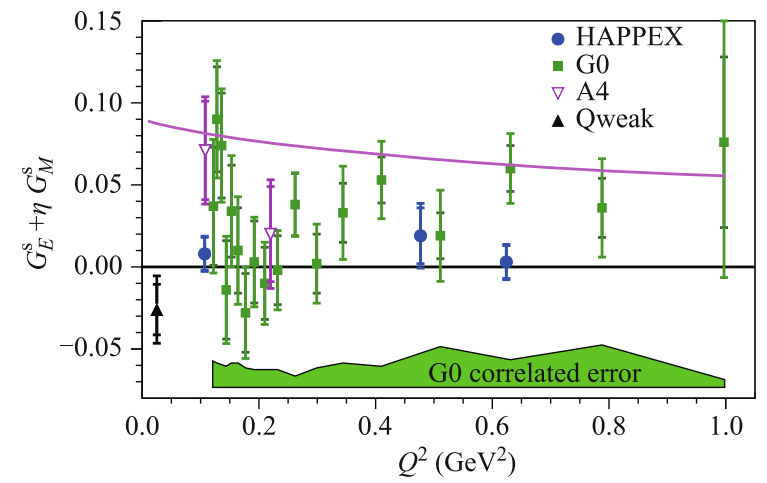

Fig. 14 Results for the strange form-factor contribution $G_{E}^{s}+$ $\eta G_{M}^{s}$ implied by measurements of forward-scattering from the proton. Here, $\eta=\tau G_{M}^{p} /\left(\epsilon G_{E}^{p}\right)$ and is numerically approximately $Q^{2}$ (in units of $\mathrm{GeV}^{2} / c^{2}$ ). For comparison the pink line shows $3 \%$ of the combination $G_{E}^{p}+\eta G_{M}^{p}$.

quarks could contribute to the charge and magnetic form factors of the nucleon? This produced many theoretical papers, some of which suggested that the strange form factors could be large enough to observe in PVES experiments.

The results of such experiments $[17,22]$ are shown in Figs. 14 and 15. At $Q^{2} \sim 0.1(\mathrm{GeV} / \mathrm{c})^{2}, Q^{2} \sim 0.22$ $(\mathrm{GeV} / \mathrm{c})^{2}$, and $Q^{2}=0.62(\mathrm{GeV} / \mathrm{c})^{2}, G_{E}^{s}$ and $G_{M}^{s}$ values were determined independently by a series of measurements at different angles and various targets. There are a number of reviews of the field [84-89]. Within the errors, the data show no strong evidence for strange form factors. Lattice QCD calculations have grown increasingly robust and are consistently calculating the strange form factors to be at the level of, or significantly smaller than, the experimental constraints [90, 91].

\subsection{Electroweak tests}

The SM, which was established more or less in its present form by about 1978, has been exceptionally successful in predicting the results of a wide body of very precise experiments. Why should people search for something more?

One simple argument is that because atoms are made up of electrons and nuclei, nuclei are made up of protons and neutrons, and protons are made up of quarks, can we not expect quarks to be made up of more elementary particles? Another argument is that the universe in known to have much more dark matter than SM matter, and explaining the dark matter requires BSM physics.

In addition, the SM has some problems. The main one is called the hierarchy problem, which is essentially that radiative corrections should make the Higgs particle much more massive than it is observed to be. Another argument is that supersymmetry (SUSY) is a very at- 
tractive theory that solves the hierarchy problem, so why is it not realized in nature? Alternatively, compositeness theories can solve the hierarchy problem.

Finally, a few experiments are inconsistent with the SM. A prime example is the muon $g-2$ experiment, which observes $\Delta a_{\mu}=a_{\mu}^{e x p}-a_{\mu}^{S M}=287(80) \times 10^{11}$, a $3.8 \sigma$ deviation from the SM. The results from the $\mathrm{NuTeV}$
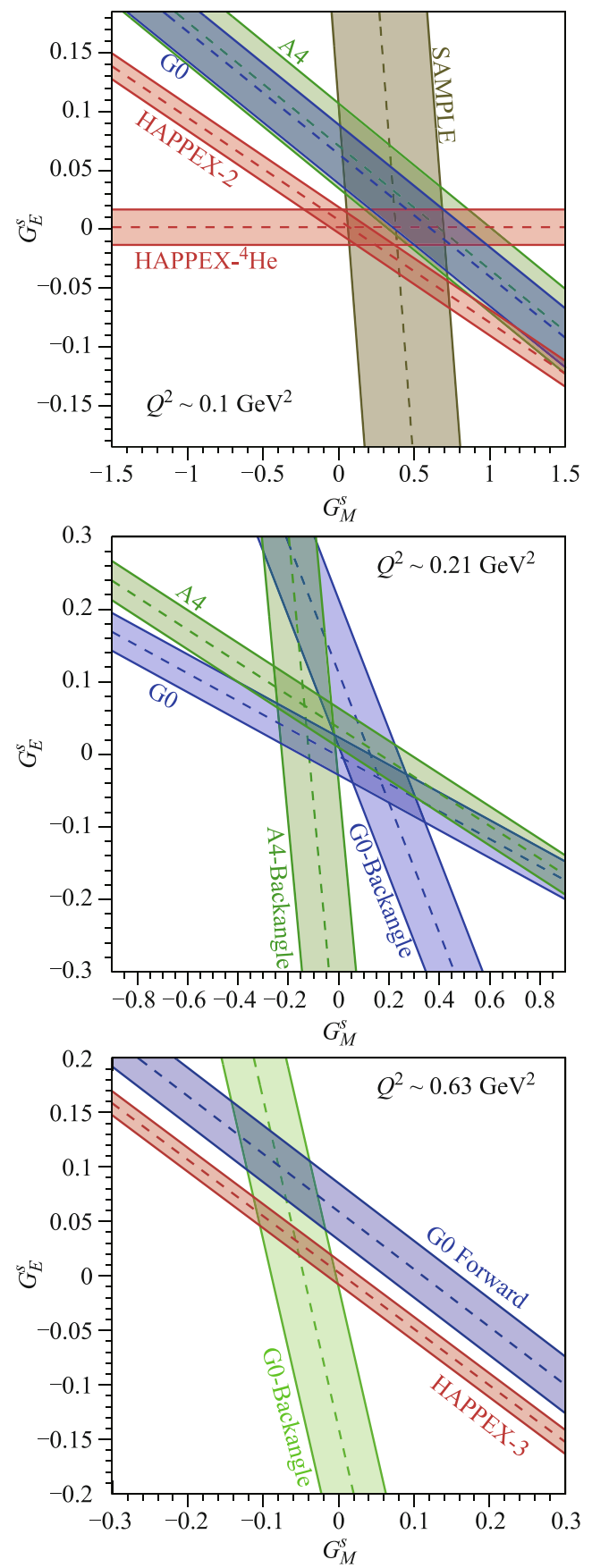

Fig. 15 1- $\sigma$ uncertainty bands corresponding to constraints on $G_{E}^{s}$ and $G_{M}^{s}$ implied by measurements of parity violation in elastic scattering from the proton and from the ${ }^{4}$ He nucleus. The axial form factors include radiative correction [41]. Uncertainties in the non-strange form factors are not reflected in these plots. experiment are another example.

There are two general approaches to searching for BSM physics: experiments at high energies and precision measurements. High-energy data have been obtained from various colliders, including LEP (an $e^{+}-e^{-}$collider), HERA (an $e^{ \pm}-p$ collider), and Fermilab and the LHC (hadron colliders). PVES, APV, and the muon $g-2$ experiments are examples of precision measurements. Both approaches have advantages and disadvantages, but in general they are complementary.

One example of a task for which high-energy colliders, especially the LHC, are ideal is the search for new $Z$ bosons $[92,93]$ that have significant branching ratios to leptons. Owing to the high rates at the LHC, it is difficult to trigger on and sift through all of the potentially interesting events. However, events with high-energy leptons are easy to trigger on efficiently, and the rates for such events are manageable. Moreover, the signal appears as a bump at a specific mass, so the discovery provides both a specific mass plus information on the production rate times the decay rate.

Low-energy experiments, such as the muon $g-2$ one, have a quite different sensitivity. Its discrepancy from the SM is sensitive to many possible BSM scenarios, such as heavy SUSY particles or low-mass "dark" photons. However, other experiments are required to select the correct explanation.

A strength of PVES is that it measures an interference. If there is BSM physics with amplitude $T_{B S M}$, it will interfere with the electromagnetic amplitude $T_{\gamma}$, and the result will be

$$
A_{P V} \propto \frac{\left|T_{\gamma}+T_{B S M}\right|^{2}-\left|T_{\gamma}-T_{B S M}\right|^{2}}{2 T_{\gamma}^{2}} \sim \frac{T_{B S M}}{T_{\gamma}} .
$$

Both amplitudes are real. In contrast, an amplitude $T_{Z}$ on the $Z$ pole is imaginary, so

$$
\sigma \propto\left|T_{Z}+T_{B S M}\right|^{2}=\left|T_{Z}\right|^{2}\left[1+\left(\frac{T_{B S M}}{T_{Z}}\right)^{2}\right],
$$

and the sensitivity to $T_{B S M}$ is suppressed because it is a small number squared. On the other hand, the highprecision $Z$ pole data provide the best limit so far on radiative corrections due to possible new physics.

A special feature of PVES searches is that if an effect is seen, it must violate parity. In addition, depending on the reaction, the couplings to the electrons and to each quark flavor can be determined.

The values of the measured asymmetries and their corresponding $Q^{2}$ for recent PVES experiments testing the SM are given in Table 5 . There is a wide range in the 
Table 5 Measured asymmetries for recent PVES experiments testing the SM. There is a large range in the values for $A_{P V}$.

\begin{tabular}{ccccc}
\hline Experiment & $\left\langle Q^{2}\right\rangle(\mathrm{GeV} / \mathrm{c})^{2}$ & $A_{P V}(\mathrm{ppm})$ & $\sigma($ Stat. $)$ & $\sigma$ (Syst.) \\
\hline SLAC E158 & 0.026 & -0.131 & 0.114 & 0.010 \\
\hline $\begin{array}{c}\text { Qweak } \\
\text { (commisioning) }\end{array}$ & 0.025 & -0.280 & 0.035 & 0.031 \\
\hline JLab PVDIS & 1.09 & 91.1 & 3.1 & 3.0 \\
\hline JLab PVDIS & 1.90 & 160.8 & 6.4 & 3.1 \\
\hline
\end{tabular}

Table 6 Values of $Q_{W}$ or coupling constants for experiments testing the SM. For Cs, we have divided the nuclear $Q_{W}$ by the number of nucleons so the various values can more easily be compared

\begin{tabular}{cccc}
\hline Experiment & Quantity & Result & SM theory \\
\hline Cs APV & $Q_{W}(\mathrm{Cs}) / 133$ & $-0.546 \pm 0.003$ & 0.551 \\
\hline SLAC E158 & $Q_{W}(e)$ & $0.037 \pm 0.05$ & 0.045 \\
\hline $\begin{array}{c}\text { elastic } \\
\text { nuclear PVES }\end{array}$ & $Q_{W}(p)$ & $0.064 \pm 0.012$ & 0.071 \\
\hline JLab PVDIS & $2 C_{2 u}-C_{2 d}$ & $-0.145 \pm 0.068$ & -0.095 \\
\hline
\end{tabular}

values of the measured asymmetries because of both the wide range in $Q^{2}$ and also the fact that the PVDIS asymmetries are not suppressed by a $\left(1-4 \sin ^{2} \theta_{W}\right)$ factor. The relevant electroweak coupling constants extracted from the asymmetries are given in Table 6 . There are several interesting points. The effect of the radiative corrections on $Q_{W}(e)$ and $Q_{W}(p)$ (see Section 2.3) is confirmed. The value of $2 C_{2 u}-C_{2 d}$ is measured for the first time to be nonzero, as predicted by the SM.

\subsubsection{Limits on compositeness}

If there is BSM physics due to interactions at short distance scales, there may be new contact interactions such that the parity-violating Lagrangian has the form

$$
\mathcal{L}=\left[\frac{G_{F}}{\sqrt{2}} C_{1 q}^{S M}+\frac{4 \pi}{\left(\Lambda_{A V}^{e q}\right)^{2}}\right] \bar{e} \gamma^{\mu} \gamma^{5} e \bar{q} \gamma_{\mu} q+\ldots
$$

where the $\Lambda_{A V}^{e q}$ term has the same Lorentz structure as the $C_{1 q}$ term, and so on.

This form [94] is inspired by models in which quarks and leptons are composed of more fundamental, strongly interacting particles characterized by a compositeness scale $\Lambda_{A V}^{e q}$ and where the coupling constant is $4 \pi$. BSM physics for such models does not have signatures such as lepton pairs that are easy to separate from the background at the LHC, so PVES experiments can set competitive limits.

If the result of a PVES experiment indicates a coupling constant that differs by $\Delta C_{i j}$ from that predicted by the SM, it can be attributed to a term in Eq. (40) with

$$
\Lambda_{A V}^{e q}=v \sqrt{\frac{8 \pi}{\left|C_{i j}\right|}},
$$

where $v=\left(\sqrt{2} G_{F}\right)^{-1 / 2}$ is the vacuum expectation value of the Higgs field. If the data are consistent with the SM, we define $\left|\Delta C_{i j}^{ \pm}\right| \equiv\left|C_{i j}^{e x p}-C_{i j}^{S M} \pm \hat{\sigma}\right|$ and

$$
\Lambda_{ \pm}>v \sqrt{\frac{8 \pi}{\left|\Delta C_{i j}^{ \pm}\right|}},
$$

where $\hat{\sigma}$ is the error corresponding to the $95 \%$ confidence level.

For experiments that measure more complicated Lorentz and isospin structure, it is appropriate to rotate the operators as explained in Ref. [6]. For the Qweak experiment, for example, the appropriate part of the Lagrangian is

$$
\left[\left(\frac{2 C_{1 u}+C_{1 d}}{\sqrt{5}}\right)+\frac{4 \pi}{\left(\Lambda_{A V}^{p}\right)^{2}}\right] \frac{\bar{e} \gamma^{\mu} \gamma^{5} e}{2 v^{2}}\left(\frac{2 \bar{u} \gamma_{\mu} u+\bar{e} \gamma_{\mu} d}{\sqrt{5}}\right) .
$$

Then

$$
\Lambda_{A V}^{p}>v \sqrt{\frac{8 \sqrt{5} \pi}{\left|\left(2 C_{2 u}+C_{2 d}\right)^{ \pm}\right|}} .
$$

Figure 16 is a plot showing the mass limits obtained from Qweak and PVDIS for both published and projected results.

\subsubsection{Leptophobic Z' boson}

It is possible that new physics exists at a somewhat lower mass scale, at the level of $100 \mathrm{GeV}$ or so, that has yet to be detected because of its weak signature in hadron colliders. One example is the hypothetical leptophobic $Z^{\prime}$

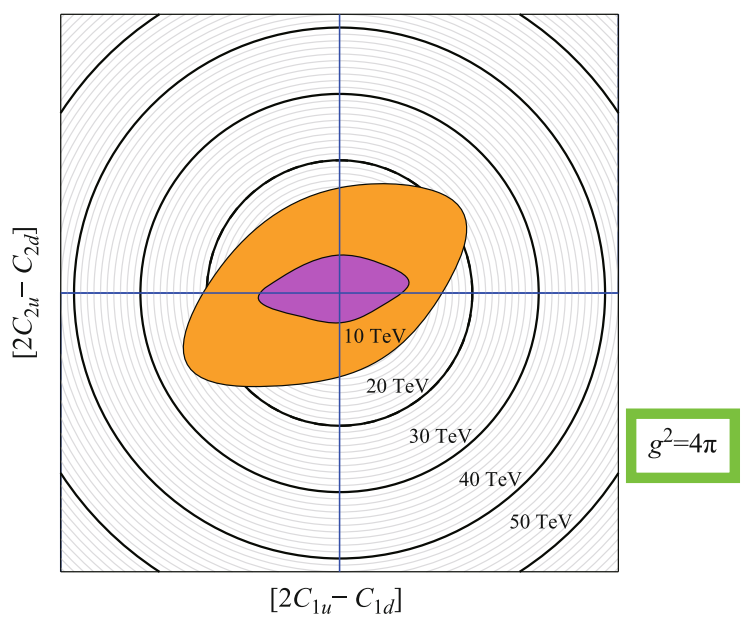

Fig. 16 Projected limits for composite models. Magenta region is excluded by published data. Orange region can be excluded with the SoLID PVES experiment and the final Qweak results. 


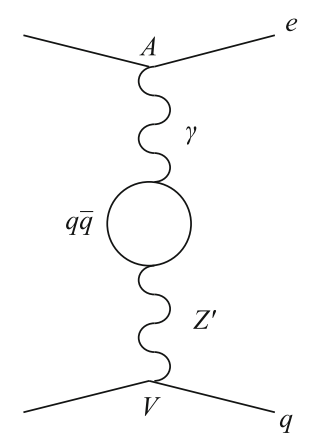

Fig. 17 Diagram fort the leptophobic $Z^{\prime}$. It is quite similar to the diagram in Fig. 2 except that the $V$ and $A$ vertices are reversed and the weak particle is the $Z^{\prime}$ instead of the $Z$.

boson, which couples mainly to quarks. In terms of lowenergy experiments, the leptophobic $Z^{\prime}$ has the feature that it couples to electrons through quark loops that connect to photons (see Fig. 17), so the coupling is strictly vector. Consequently, it has no effect on the $C_{1}$ values and can be observed only in PV experiments that are sensitive to the $C_{2}$ values, such as SoLID-PVDIS [95, 96].

One specific model, which was developed to explain excess di-quark production observed at Fermilab in the $100 \mathrm{GeV}$ mass region [97], is based on the $E(6)$ group. An interesting feature of this model that distinguishes it from older versions is that it also couples to dark matter and is relevant to dark matter experiments [98]. For example, the $Z^{\prime}$ could be the particle exchanged in experiments designed to detect dark matter. An interesting point is that for such a low-mass $Z^{\prime}$, the Fermilab data are more sensitive than the LHC data. For some mass ranges, the signal of this $Z^{\prime}$ would be lost in the background in any collider.

\subsubsection{Dark light}

It is possible that BSM physics could involve a new lowmass particle. A classic example is the axion, which can explain why parity is conserved in strong interactions [99]. A light particle that can cause party violation was proposed by Fayet $[100,101]$ some time ago. More recently, there has been considerable interest in a parityconserving low mass "dark" photon [102] that couples to both SM and dark matter through kinetic mixing. The mass of the dark photon is well below that of the SM $Z$, perhaps as low as a few mega-electron volts or less, and it has a weak coupling to ordinary matter given by the small parameter $\varepsilon$.

If, in addition, the dark photon and the SM $Z$ boson undergo mass mixing characterized by the small parameter $\delta$, the interaction can be parity violating $[103,104]$.
The existence of such a particle, called the dark $Z$, modifies the parameters $\hat{\kappa}_{e}$ and $\hat{\kappa}_{e}^{\prime}$ from Table 2 as follows:

$$
\begin{aligned}
& \hat{\kappa}_{e}^{(\prime)} \rightarrow \hat{\kappa}_{e}^{(\prime)}-\varepsilon \delta \frac{M_{Z}}{M_{Z_{d}}} \cot \theta_{W} \frac{m_{Z_{d}}^{2}}{Q^{2}+m_{Z_{d}}^{2}}, \\
& \hat{\rho}_{e}^{(\prime)} \rightarrow \hat{\rho}_{e}^{(\prime)}+\delta^{2} \frac{M_{Z}}{M_{Z_{d}}} \frac{m_{Z_{d}}^{2}}{Q^{2}+m_{Z_{d}}^{2}} .
\end{aligned}
$$

The $\hat{\kappa}$ term causes $Q_{W}(e)$ and $Q_{W}(p)$ to differ from the SM predictions at values of $Q^{2}$ near and below $m_{Z_{d}}$. For $Q_{W}(C s)$, both $\hat{\rho}$ and $\hat{\kappa}$ may contribute. It is even possible that the two terms cancel.

There are a number of possible experimental signatures for these new particles. In some models, the dark photon decays to an electron-positron pair with a fixed mass, providing a clean signature. In other cases, the dark photon decays to invisible particles but can be seen in the decay $K^{+} \rightarrow \pi Z_{d}$. Indirect effects, such as PVES or $\Delta a$ for the $g-2$ experiment, are independent of the decay modes. The effect of the dark $Z$ is enhanced by the ratio $M_{Z} / M_{Z_{d}}$, which makes PVES competitive with other experiments. PVES is independent of whether the $Z_{d}$ decays into SM or invisible particles or whether $m_{Z_{D}}$ is near the mass of the $\pi^{0}$, in which case other searches are swamped by the background.

\subsubsection{SUSY}

Theories that invoke SUSY also can affect PVES, although they can be observed only by the most sensitive experiments [105]. For conventional SUSY, which conserves a symmetry called $R$ parity, the main effects are due to loops. For R-parity-violating SUSY theories, treelevel contributions are possible. The R-parity-violating theories are less attractive because they do not provide a stable particle that could be the component of dark matter.

\subsection{Radius of neutron distributions}

The physics of neutron-rich matter, where $N \gg Z$, affects a number of fields. Among the more interesting is that of neutron stars, where the immense force of gravity causes protons and electrons to combine to make neutrons in order to lower the electrostatic energy. However, the extra neutrons are accompanied by an increase in the nuclear symmetry energy, which is defined by the equation

$$
\frac{E}{A}\left(\rho_{n} \neq \rho_{p}\right) \approx \frac{E}{A}\left(\rho_{n}=\rho_{p}\right)+\frac{\rho_{n}-\rho_{p}}{\rho_{n}-\rho_{p}} S(\rho) .
$$

The symmetry energy has two sources, kinetic and po- 
Table 7 Achieved (upper panel) or anticipated (middle panel) relative uncertainty, the corresponding tree level sensitivity to extract the weak mixing angle, and the expected reach to the associated compositeness scales (at 95\% CL) for key parity-violation experiments. The lower panel gives limits on compositeness from various collider experiments including Atlas [106] and CMS [107] at the LHC, the H1 experiment at HERA [108], and the OPAL experiment at LEP [109].

\begin{tabular}{cccc}
\hline & Precision $(\%)$ & $\Delta \sin ^{2} \hat{\theta}_{W}(0)$ & $\Lambda_{\text {new }}(\mathrm{TeV})$ \\
\hline SLAC-E122 & 8.3 & 0.011 & 5.3 \\
APV $\left({ }^{133} \mathrm{Cs}\right)$ & 0.58 & 0.0019 & 32.3 \\
SLAC-E158 & 14 & 0.0013 & 17.0 \\
JLab-Qweak & 19 & 0.0030 & 17.0 \\
JLab-Hall A & 4.1 & 0.0051 & 7.8 \\
\hline JLab-Qweak (final) & 4.5 & 0.0008 & 33 \\
JLab-SoLID & 0.6 & 0.00057 & 22 \\
JLab-MØLLER & 2.3 & 0.00026 & 39 \\
Mainz-P2 & 2.0 & 0.00036 & 49 \\
APV ( ${ }^{225}$ Ra $\left.{ }^{+}\right)$ & 0.5 & 0.0018 & 34 \\
PVES (12C) & 0.3 & 0.0007 & 49 \\
\hline LEP (OPAL) & & & $3-16$ \\
HERA (H1) & & & $3.6-7.2$ \\
ATLAS di-leptons & & & $8.8-15$ \\
CMS jets & &
\end{tabular}

tential energy. The kinetic energy arises because nuclear matter is a degenerate Fermi gas. Identical fermions must be in different quantum states, not just in the states with the lowest kinetic energy. The strong force of the nucleons is stronger for neutron-proton pairs because the force is short-range and is stronger for different particles that can be in the same state.

Nuclear systems thus balance the Coulomb and symmetry energies. For light nuclei, where the Coulomb energy is small, $N=Z$. The heavy nucleus ${ }^{208} \mathrm{~Pb}$ has $40 \%$ protons, but neutron stars are made up of only about $10 \%$ protons.

An important parameter for neutron-rich systems is the density dependence of the symmetry energy:

$$
L=\left.3 \rho_{0} \frac{\mathrm{d} S}{\mathrm{~d} \rho}\right|_{\rho_{0}} .
$$

For heavy nuclei, there is a core with a radius of about $5.5 \mathrm{fm}$ and a uniform density $\rho_{0} \approx 0.16 \mathrm{fm}^{-3}$, and a "skin" of lower density extending to about $8 \mathrm{fm}$. If the symmetry energy rises rapidly with the density, the extra neutrons in $\mathrm{Pb}$ would decrease their energy by moving to the skin and increase $\Delta R=R_{n}-R_{p}$. Indeed, in this case, the skin of a heavy nucleus is a site of neutron-rich matter. It is this same pressure that supports neutron stars. Measuring $\Delta R$ using PVES is probably the most reliable way to determine $L$. There is a strong correlation between $\Delta R^{208}$ and the pressure $P$ of nuclear matter at densities equal to about $2 / 3$ of that at the center of heavy nuclei.

A detailed description of bulk nuclear matter, including heavy nuclei as well as the nuclear material in neutron stars, is given by density functional theory (DFT). DFT is a quantum mechanical theory based on an energy functional. A large body of nuclear data, including nuclear sizes, shapes, and energy levels, is used to adjust the parameters of DFT. This constrains the nuclear equation of state (EOS), or the pressure of nuclear matter as a function of the density. However, most of the input is insensitive to the properties of neutron-rich matter, so data on $\Delta R$ greatly affect predictions of the EOS for neutron-rich systems [110].

Neutron stars are complex objects in which neutronrich matter is present in many different phases, including the liquid, solid, superfluid, and liquid crystal phases, because the pressure due to gravity depends strongly on the depth. The central density of a neutron star is $\sim 5-10$ times $\rho_{0}$, but it is closer to that of ordinary nuclear matter near the surface. The value of $L$ has a direct impact on determining the properties at the surface of neutron stars and can also be used to extrapolate the symmetry energy at higher central densities.

Many observables are possible for neutron stars, especially with the advent of X-ray satellites such as Chandra and the expectation of observations of gravity waves from neutron stars by LIGO.

The mass of neutron stars can be determined precisely from observations of binary pulsars. Neutron stars have been observed to have masses between the Chandrasekhar limit of 1.4 solar masses and about 2 solar masses. Larger values of $L$ would suggest a stiffer EOS and allow for even larger neutron star masses.

The radius $R_{1}$ of neutron stars is expected to be in the range of 8-11 km. Larger values of $\Delta R$ from ${ }^{208} \mathrm{~Pb}$, such as that suggested by the PREx-I result, would imply a stiffer EOS and larger values of $R_{1}$. It is anticipated that $R_{1}$ can be measured by luminosity and spectral measurements by an X-ray satellite. The basic idea is that the luminosity of an object is given by the Stefan-Boltzmann law,

$$
\mathcal{L}=4 \pi R_{1}^{2} \sigma T^{4} .
$$

The temperature can be extracted from the X-ray energy spectrum. There are a number of corrections. For example, the effective radius $R_{1}$ of a neutron is larger than the actual radius $R$,

$$
R_{1}=\frac{R}{1-2 G M / R}
$$

owing to the effects of curved space in general relativity. Here $G$ is the gravitational constant. The distance of 
the star must be known, which is possible if it is located in a known globular cluster. Further, the effects of the atmosphere of the star on the X-ray spectrum must be known.

The advanced LIGO gravitational wave observatory [111] is expected to make the first direct observations of gravity waves in the near future [112]. Two types of signals arise from neutron stars:

1) Pulsar-type signals from rotating neutron stars;

2) "Chirp" signals from the merging or in-spiraling of a binary system with a neutron star.

The EOS is critical for both observations. For a rotating neutron star to emit gravity waves, it must have a significant quadrupole moment. If $\Delta R$ for ${ }^{208} \mathrm{~Pb}$ is large enough, the solid crust of the star might be stiff enough [113] to support "mountains" of sufficient size.

The probability of observing merging neutron stars is proportional to the number of neutron stars present in galaxies. Only hot neutron stars are directly observable, so the total number of neutron stars depends on how fast they cool and thus what fraction are observed. Neutron stars cool through neutrino emission in the socalled URCA process. The rate of URCA cooling depends strongly on the level of the Fermi sea of the protons in the star, which depends in turn on the symmetry energy. If the Fermi level is too high, neutrinos can be emitted only by a slow three-body process. The proton fraction in the core of the star, which determines the rate of the URCA cooling process, is correlated with $\Delta R$ [114]. Thus, a measurement of $\Delta R$ can help predict how many signals will be observed.

Two neutron-rich nuclei are currently of interest, ${ }^{208} \mathrm{~Pb}$, which has 44 extra neutrons, and ${ }^{48} \mathrm{Ca}$, which is much lighter but still has 8 extra neutrons. The ${ }^{48} \mathrm{Ca}$ nucleus is of special interest because its properties can be computed both by DFT and also by coupled cluster calculations [115], which are based on nucleon forces instead of bulk nuclear properties. Coupled cluster calculations thus have the potential to provide information on the possibility of three-neutron forces.

Isospin diffusion in heavy ion collisions, an important topic for exotic nuclei, depends strongly on $L$. The neutron skin also contributes to the asymmetry measured in APV experiments. If a new generation of experiments is performed, the PVES data will be important for making this correction.

The PREx experiment measured [29]

$$
A_{P V}=0.656 \pm 0.060(\text { stat }) \pm 0.014(\text { syst }) \text {, }
$$

which implies that $\Delta R^{208}=0.33_{-0.18}^{+0.16} \mathrm{fm}$. The goal of the program is to reach a precision of $0.06 \mathrm{fm}$.

\section{$5 \quad$ Summary and conclusions}

Since the pioneering work of Prescott et al. in 1978, the field of PVES has made dramatic progress. In addition to providing a test of the SM, PVES also yields unique information on the features of QCD, including strangeness in the nucleon, CSV, and the properties of HT effects. In addition, PVES probes key aspects of the properties of nuclear systems, including neutron stars. Over the years, the sensitivity of experiments has improved dramatically, as is clearly seen in Fig. 1. We are awaiting the next generation of PVES experiments, including the P2, PREx, CREx, MOLLER, and SoLID experiments.

Acknowledgements P.A.S. is funded in part by the Department of Energy (DOE) under grants DE-FG02-84ER40146 (PAS) and DE-FG02-07ER41522 (KDP). We thank K. de Jager for carefully reading the manuscript and making many helpful suggestions.

Open Access This article is distributed under the terms of the Creative Commons Attribution License which permits any use, distribution, and reproduction in any medium, provided the original author(s) and the source are credited.

\section{References}

1. T. Donnelly and J. Walecka, Electron scattering and nuclear structure, Annu. Rev. Nucl. Part. Sci. 25(1), 329 (1975)

2. E. D. Bloom, D. Coward, H. DeStaebler, J. Drees, G. Miller, L. W. Mo, R. E. Taylor, M. Breidenbach, J. I. Friedman, G. C. Hartmann, and H. W. Kendall, High-energy inelastic e-p scattering at $6^{\circ}$ and $10^{\circ}$, Phys. Rev. Lett. 23(16), 930 (1969)

3. M. Breidenbach, J. I. Friedman, H. W. Kendall, E. D. Bloom, D. H. Coward, H. DeStaebler, J. Drees, L. W. Mo, and R. E. Taylor, Observed behavior of highly inelastic electron-proton scattering, Phys. Rev. Lett. 23(16), 935 (1969)

4. K. Kumar, S. Mantry, W. Marciano, and P. Souder, Lowenergy measurements of the weak mixing angle, Annu. Rev. Nucl. Part. Sci. 63(1), 237 (2013)

5. M. J. Ramsey-Musolf, Low-energy parity-violation and new physics, Phys. Rev. C 60(1), 015501 (1999)

6. J. Erler, C. J. Horowitz, S. Mantry, and P. A. Souder, Weak polarized electron scattering, Annu. Rev. Nucl. Part. Sci. 64(1), 269 (2014)

7. C. S. Wu, E. Ambler, R. W. Hayward, D. D. Hoppes, and R. P. Hudson, Experimental test of parity conservation in beta decay, Phys. Rev. 105(4), 1413 (1957)

8. C. Y. Prescott, et al. (SLAC-E122 Collaboration), Parity non-conservation in inelastic electron scattering, Phys. Lett. 


\section{B 77, 347 (1978)}

9. C. Y. Prescott, et al. (SLAC-E122 Collaboration), Further measurements of parity non-conservation in inelastic electron scattering, Phys. Lett. B 84, 524 (1979)

10. W. Heil, J. Ahrens, H. G. Andresen, A. Bornheimer, D. Conrath, K. J. Dietz, W. Gasteyer, H. J. Gessinger, W. Hartmann, J. Jethwa, H. J. Kluge, H. Kessler, T. Kettner, L. Koch, F. Neugebauer, R. Neuhausen, E. W. Otten, E. Reichert, F. P. Schäfer, and B. Wagner, Improved limits on the weak, neutral, hadronic axial vector coupling constants from quasielastic scattering of polarized electrons, Nucl. Phys. B 327(1), 1 (1989)

11. P. A. Souder, R. Holmes, D. Kim, K. S. Kumar, M. E. Schulze, K. Isakovich, G. W. Dodson, K. W. Dow, M. Farkhondeh, S. Kowalski, M. S. Lubell, J. Bellanca, M. Goodman, S. Patch, R. Wilson, G. D. Cates, S. Dhawan, T. J. Gay, V. W. Hughes, A. Magnon, R. Michaels, and H. R. Schaefer, Measurement of parity violation in the elastic scattering of polarized electrons from ${ }^{12} \mathrm{C}$, Phys. Rev. Lett. 65(6), 694 (1990)

12. J. Ashman, et al. (The European Muon Collaboration), A measurement of the spin asymmetry and determination of the structure function $g_{1}$ in deep inelastic muon-proton scattering, Phys. Lett. B 206, 364 (1988)

13. J. Ashman, et al. (The European Muon Collaboration), An investigation of the spin structure of the proton in deep inelastic scattering of polarised muons on polarised protons, Nucl. Phys. B 328, 1 (1989)

14. D. B. Kaplan and A. Manohar, Strange matrix elements in the proton from neutral-current experiments, Nucl. Phys. B 310(3-4), 527 (1988)

15. R. McKeown, Sensitivity of polarized elastic electron-proton scattering to the anomalous baryon number magnetic moment, Phys. Lett. B 219(2-3), 140 (1989)

16. D. T. Spayde, et al. (SAMPLE Collaboration), Parity violation in elastic electron-proton scattering and the proton's strange magnetic form factor, Phys. Rev. Lett. 84, 1106 (2000)

17. T. Ito, et al. (SAMPLE Collaboration), Parity-violating electron deuteron scattering and the proton's neutral weak axial vector form factor, Phys. Rev. Lett. 92, 102003 (2004)

18. D. T. Spayde, et al. (SAMPLE Collaboration), The strange quark contribution to the proton's magnetic moment, Phys. Lett. B 583, 79 (2004)

19. K. A. Aniol, et al. (HAPPEX Collaboration), Parityviolating electroweak asymmetry in ep scattering, Phys. Rev. C 69, 065501 (2004)

20. A. Acha, et al. (HAPPEX Collaboration), Precision measurements of nucleon strange form factors at $Q^{2} \sim 0.1 \mathrm{GeV}^{2}$, Phys. Rev. Lett. 98, 032301 (2007)

21. Z. Ahmed, et al. (HAPPEX Collaboration), New precision limit on the strange vector form factors of the proton, Phys. Rev. Lett. 108, 102001 (2012)
22. D. S. Armstrong, et al. (G0 Collaboration), Strange-quark contributions to parity-violating asymmetries in the forward G0 electron-proton scattering experiment, Phys. Rev. Lett. 95, 092001 (2005)

23. D. Androic, et al. (G0 Collaboration), Strange quark contributions to parity-violating asymmetries in the backward angle G0 electron scattering experiment, Phys. Rev. Lett. 104, 012001 (2010)

24. F. Maas, et al. (A4 Collaboration), Measurement of strange-quark contributions to the nucleon's form factors at $Q_{2}=0.230(\mathrm{GeV} / \mathrm{c})^{2}$, Phys. Rev. Lett. 93, 022002 (2004)

25. F. E. Maas, K. Aulenbacher, S. Baunack, L. Capozza, J. Diefenbach, B. Gläser, T. Hammel, D. von Harrach, Y. Imai, E. M. Kabuss, R. Kothe, J. H. Lee, A. Lorente, E. Schilling, D. Schwaab, M. Sikora, G. Stephan, G. Weber, C. Weinrich, I. Altarev, J. Arvieux, M. El-Yakoubi, R. Frascaria, R. Kunne, M. Morlet, S. Ong, J. van de Wiele, S. Kowalski, B. Plaster, R. Suleiman, and S. Taylor, Evidence for strange-quark contributions to the nucleon's form factors at $Q_{2}=0.108(\mathrm{GeV} / c)^{2}$, Phys. Rev. Lett. 94(15), 152001 (2005)

26. S. Baunack, et al. (A4 Collaboration), Measurement of strange quark contributions to the vector form factors of the proton at $Q_{2}=0.22(\mathrm{GeV} / c)^{2}$, Phys. Rev. Lett. 102(15), 151803 (2009)

27. P. L. Anthony, et al. (SLAC E158 Collaboration), Precision measurement of the weak mixing angle in Møller scattering, Phys. Rev. Lett. 95, 081601 (2005)

28. T. Allison, et al. (Qweak Collaboration), The $Q_{\text {weak }}$ experimental apparatus, Nucl. Instrum. Methods A 781, 105 (2015)

29. S. Abrahamyan, et al. (PREX Collaboration), Measurement of the neutron radius of ${ }^{208} \mathrm{~Pb}$ through parity violation in electron scattering, Phys. Rev. Lett. 108, 112502 (2012)

30. D. Becker, et al. (P2 Collaboration), P2 - The weak charge of the proton, PoS Bormio 2013: 024 (2013)

31. J. Benesch, et al. (MOLLER Collaboration), The MOLLER experiment: An ultra-precise measurement of the weak mixing angle using Møller scattering, arXiv: 1411.4088 [nucl-ex]

32. J. Formaggio and G. Zeller, From eV to EeV: Neutrino cross sections across energy scales, Rev. Mod. Phys. 84(3), 1307 (2012)

33. G. P. Zeller, et al. (NuTeV Collaboration), Precise determination of electroweak parameters in neutrino-nucleon scattering, Phys. Rev. Lett. 88, 091802 (2002)

34. C. S. Wood, S. C. Bennett, D. Cho, B. P. Masterson, J. L. Roberts, C. E. Tanner, and C. E. Wieman, Measurement of parity nonconservation and an anapole moment in cesium, Science 275(5307), 1759 (1997)

35. G. S. Giri, O. O. Versolato, L. W. Wansbeek, J. E. van den Berg, D. J. van der Hoek, K. Jungmann, W. L. Kruithof, C. J. G. Onderwater, B. K. Sahoo, B. Santra, P. D. Shidling, R. G. E. Timmermans, L. Willmann, and H. W. Wilschut, Precision spectroscopy of trapped radioactive radium ions, Can. J. Phys. 89(1), 69 (2011) 
36. H. Frauenfelder and E. Henley, Subatomic Physics, NY: John Wiley and Sons, 1974

37. O. Moreno, T. Donnelly, R. Gonzalez-Jimenez, and J. Caballero, Evaluation of theoretical uncertainties in parityviolating electron scattering from nucleons and nuclei, $J$. Phys. G 42(3), 034006 (2015)

38. T. Donnelly, J. Dubach, and I. Sick, Isospin dependences in parity-violating electron scattering, Nucl. Phys. A. 503(34), 589 (1989)

39. M. J. Musolf and B. R. Holstein, Electroweak corrections to parity-violating neutral current scattering, Phys. Lett. B 242(3-4), 461 (1990)

40. A. Czarnecki and W. J. Marciano, Electroweak radiative corrections to polarized Møller scattering asymmetries, Phys. Rev. D 53(3), 1066 (1996)

41. S. L. Zhu, S. J. Puglia, B. R. Holstein, and M. J. RamseyMusolf, Nucleon anapole moment and parity-violating ep scattering, Phys. Rev. D 62(3), 033008 (2000)

42. N. Hall, P. Blunden, W. Melnitchouk, A. Thomas, and R. Young, Hadronic $\gamma Z$ box corrections in Møller scattering, Phys. Lett. B 731, 287 (2014)

43. M. Gorchtein, C. Horowitz, and M. J. Ramsey-Musolf, Model dependence of the $\gamma Z$ dispersion correction to the parity-violating asymmetry in elastic ep scattering, Phys. Rev. C 84(1), 015502 (2011)

44. D. H. Beck, Strange-quark vector currents and parityviolating electron scattering from the nucleon and from nuclei, Phys. Rev. D 39(11), 3248 (1989)

45. C. J. Horowitz, Parity violating elastic electron scattering and Coulomb distortions, Phys. Rev. C 57(6), 3430 (1998)

46. C. J. Horowitz, S. J. Pollock, P. A. Souder, and R. Michaels, Parity violating measurements of neutron densities, Phys. Rev. C 63(2), 025501 (2001)

47. T. Hobbs and W. Melnitchouk, Finite- $Q_{2}$ corrections to parity-violating DIS, Phys. Rev. D 77(11), 114023 (2008)

48. G. R. Farrar and D. R. Jackson, Pion and nucleon structure functions near $x=1$, Phys. Rev. Lett. 35(21), 1416 (1975)

49. J. T. Londergan, J. C. Peng, and A. W. Thomas, Charge symmetry at the partonic level, Rev. Mod. Phys. 82(3), 2009 (2010)

50. W. Bentz, I. C. Cloët, J. T. Londergan, and A. W. Thomas, Reassessment of the $\mathrm{NuTeV}$ determination of the weak mixing angle, Phys. Lett. B 693(4), 462 (2010)

51. M. Glück, P. Jimenez-Delgado, and E. Reya, Radiatively generated isospin violations in the nucleon and the $\mathrm{NuTeV}$ anomaly, Phys. Rev. Lett. 95(2), 022002 (2005)

52. A. D. Martin, R. G. Roberts, W. J. Stirling, and R. S. Thorne, Parton distributions incorporating QED contributions, Eur. Phys. J. C 39(2), 155 (2005)

53. C. Boros, F. M. Steffens, J. T. Londergan, and A. W. Thomas, A new analysis of charge symmetry violation in parton distributions, Phys. Lett. B 468(1-2), 161 (1999)
54. J. D. Bjorken, Model-independent remarks on electronquark parity-violating neutral-current couplings, Phys. Rev. D 18(9), 3239 (1978)

55. L. Wolfenstein, Testing the Weinberg-Salam model in polarized ed and ep deep inelastic scattering, Nucl. Phys. B 146(2), 477 (1978)

56. S. Mantry, M. J. Ramsey-Musolf, and G. F. Sacco, Examination of higher-order twist contributions in parity-violating deep-inelastic electron-deuteron scattering, Phys. Rev. C 82(6), 065205 (2010)

57. C. Y. Seng and M. J. Ramsey-Musolf, Parity-violating deepinelastic electron-deuteron scattering: Higher twist and parton angular momentum, Phys. Rev. C 88(1), 015202 (2013)

58. J. Qiu, Twist-4 contributions to the hadron structure functions, Phys. Rev. D 42(1), 30 (1990)

59. E. Stein, M. Maul, L. Mankiewicz, and A. Schäfer, Renormalon model predictions for power corrections to flavorsinglet deep-inelastic structure functions, Nucl. Phys. B 536(1-2), 318 (1998)

60. A. I. Signal, Calculations of higher twist distribution functions in the MIT bag model, Nucl. Phys. B 497(1-2), 415 (1997)

61. A. V. Belitsky, A. Manashov, and A. Schäfer, Twist-four corrections to parity-violating electron-deuteron scattering, Phys. Rev. D 84(1), 014010 (2011)

62. W. Melnitchouk, I. R. Afnan, F. Bissey, and A. W. Thomas, Comment on "Parton distributions, $d / u$, and higher twist effects at high $x "$, Phys. Rev. Lett. 84(23), 5455 (2000)

63. J. F. Owens, A. Accardi, and W. Melnitchouk, Global parton distributions with nuclear and finite- $Q_{2}$ corrections, Phys. Rev. D 87(9), 094012 (2013)

64. E. Derman and W. J. Marciano, Parity violating asymmetries in polarized electron scattering, Ann. Phys. 121(1-2), 147 (1979)

65. C. K. Sinclair, P. A. Adderley, B. M. Dunham, J. C. Hansknecht, P. Hartmann, M. Poelker, J. S. Price, P. M. Rutt, W. J. Schneider, and M. Steigerwald, Development of a high average current polarized electron source with long cathode operational lifetime, Phys. Rev. ST Accel. Beams 10(2), 023501 (2007)

66. P. A. Adderley, J. Clark, J. Grames, J. Hansknecht, K. Surles-Law, D. Machie, M. Poelker, M. L. Stutzman, and R. Suleiman, Load-locked dc high voltage GaAs photogun with an inverted-geometry ceramic insulator, Phys. Rev. ST Accel. Beams 13(1), 010101 (2010)

67. K. S. Kumar and P. A. Souder, Strange quarks and parity violation, Prog. Part. Nucl. Phys. 45, S333 (2000)

68. K. D. Paschke, Controlling helicity-correlated beam asymmetries in a polarized electron source, Eur. Phys. J. A 32(4), 549 (2007)

69. D. Androi et al. (Qweak Collaboration), First Determination of the Weak Charge of the Proton, Phys. Rev. Lett. 111(14), 141803 (2013) 
70. E. Beise, D. Beck, E. Candell, R. Carr, F. Duncan, T. Forest, W. Korsch, J. W. Mark, R. D. McKeown, B. A. Mueller, M. Pitt, and S. Wells, A high power liquid hydrogen target for parity violation experiments, Nucl. Instrum. Methods A 378(3), 383 (1996)

71. P. A. Souder and R. Holmes, New spectrometers for precision measurements of parity violation with polarized electrons, Proc. Parity violation in electron scattering, World Scientific, Pasadena, CA, Feb. 23-24, 1990, pp. 137-151

72. J. Alcornć, B. Anderson, K. Aniol, J. Annand, L. Auerbach, et al., Basic instrumentation for Hall A at Jefferson Lab, Nucl. Instrum. Methods A 522(3), 294 (2004)

73. D. Wang, et al. (Jefferson Lab PVDIS Collaboration), Measurement of parity violation in electron-quark scattering, Nature 506, 67 (2014)

74. D. Wang, K. Pan, R. Subedi, Z. Ahmed, K. Allada, et al., Measurement of parity-violating asymmetry in electrondeuteron inelastic scattering, Phys. Rev. C 91(4), 045506 (2015)

75. D. Androić, et al. (G0 Collaboration), The G0 experiment: Apparatus for parity-violating electron scattering measurements at forward and backward angles, Nucl. Instrum. Methods A 646(1), 59 (2011)

76. P. Achenbach, S. Baunack, K. Grimm, T. Hammel, D. vonHarrach, A. L. Ginja, F. E. Maas, E. Schilling, and H. Ströher, Measurements and simulations of Cherenkov light in lead fluoride crystals, Nucl. Instrum. Methods A 465(2-3), 318 (2001)

77. S. Baunack, et al. (A4 Collaboration), Real-time calibration of the A4 electromagnetic lead fluoride $\left(\mathrm{PbF}_{2}\right)$ calorimeter, Nucl. Instrum. Methods A 640, 58 (2011)

78. P. Souder, Parity-violating DIS at JLab with $12 \mathrm{GeV}$, in: Proceedings, 16th International Workshop on Deep Inelastic Scattering and Related Subjects (DIS 2008), Conf. Proc. C 0804071, 243 (2008)

79. P. Cooper, M. Alguard, R. Ehrlich, V. Hughes, H. Kobayakawa, J. S. Ladish, M. S. Lubell, N. Sasao, K. P. Schüler, P. A. Souder, G. Baum, W. Raith, K. Kondo, D. H. Coward, R. H. Miller, C. Y. Prescott, D. J. Sherden, and C. K. Sinclair, Polarized electron-electron scattering at $\mathrm{GeV}$ energies, Phys. Rev. Lett. 34(25), 1589 (1975)

80. L. Levchuk, The intra-atomic motion of bound electrons as a possible source of the systematic error in electron beam polarization measurements by means of a Møller polarimeter, Nucl. Instrum. Methods A 345(3), 496 (1994)

81. E. Chudakov and V. Luppov, Moller polarimetry with atomic hydrogen targets, IEEE Trans. Nucl. Sci. 51(4), 1533 (2004)

82. M. Friend, D. Parno, F. Benmokhtar, A. Camsonne, M. Dalton, G. B. Franklin, V. Mamyan, R. Michaels, S. Nanda, V. Nelyubin, K. Paschke, B. Quinn, A. Rakhman, P. Souder, and A. Tobias, Upgraded photon calorimeter with integrating readout for the Hall A Compton polarimeter at Jefferson Lab, Nucl. Instrum. Methods A 676, 96 (2012)
83. A. Narayan, et al., Precision electron-beam polarimetry using Compton scattering at $1 \mathrm{GeV}$, arXiv: 1509.06642 [nuclex]

84. R. D. Young, J. Roche, R. D. Carlini, and A. W. Thomas, Extracting nucleon strange and anapole form factors from world data, Phys. Rev. Lett. 97(10), 102002 (2006)

85. J. Liu, R. D. McKeown, and M. J. Ramsey-Musolf, Global analysis of nucleon strange form factors at low $Q_{2}$, Phys. Rev. C 76(2), 025202 (2007)

86. R. Gonzalez-Jimenez, J. A. Caballero, and T. W. Donnelly, Parity violation in elastic electron-nucleon scattering: Strangeness content in the nucleon, arXiv: 1111.6918 [nuclth]

87. K. Paschke, A. Thomas, R. Michaels, and D. Armstrong, Strange vector form factors from parity-violating electron scattering, J. Phys. Conf. Ser. 299, 012003 (2011)

88. D. S. Armstrong and R. D. McKeown, Parity-violating electron scattering and the electric and magnetic strange form factors of the nucleon, Annu. Rev. Nucl. Part. Sci. 62(1), 337 (2012)

89. R. Gonzalez-Jimenez, J. A. Caballero, and T. W. Donnelly, Global analysis of parity-violating asymmetry data for elastic electron scattering, Phys. Rev. D 90(3), 033002 (2014)

90. P. E. Shanahan, R. Horsley, Y. Nakamura, D. Pleiter, P. E. L. Rakow, G. Schierholz, H. Stüben, A. W. Thomas, R. D. Young, and J. M. Zanotti, Determination of the strange nucleon form factors, Phys. Rev. Lett. 114(9), 091802 (2015)

91. J. Green, S. Meinel, and M. Engelhardt, S. Krieg, J. Laeuchli, J. Negele, K. Orginos, A. Pochinsky, and S. Syritsyn, High-precision calculation of the strange nucleon electromagnetic form factors, Phys. Rev. D 92(3), 031501 (2015), arXiv: 1505.01803 [hep-lat]

92. P. Langacker, The physics of heavy $Z^{\prime}$ gauge bosons, Rev. Mod. Phys. 81(3), 1199 (2009)

93. T. G. Rizzo, $Z^{\prime}$ phenomenology and the LHC, arXiv: hep$\mathrm{ph} / 0610104$

94. E. Eichten, K. D. Lane, and M. E. Peskin, New tests for quark and lepton substructure, Phys. Rev. Lett. 50(11), 811 (1983)

95. M. R. Buckley and M. J. Ramsey-Musolf, Precision probes of a leptophobic $Z^{\prime}$ boson, Phys. Lett. B 712(3), 261 (2012)

96. M. Gonzalez-Alonso and M. J. Ramsey-Musolf, Leptophobic $Z^{\prime}$ boson and parity-violating eD scattering, Phys. Rev. D 87(5), 055013 (2013)

97. T. Aaltonen, et al. (CDF Collaboration), Invariant mass distribution of jet pairs produced in association with a $W$ boson in $p \bar{p}$ collisions at $\sqrt{s}=1.96 \mathrm{TeV}$, Phys. Rev. Lett. 106, 171801 (2011)

98. M. R. Buckley, D. Hooper, and J. L. Rosner, A leptophobic $Z^{\prime}$ and dark matter from grand unification, Phys. Lett. B 703(3), 343 (2011)

99. J. E. Kim and G. Carosi, Axions and the strong CP problem, Rev. Mod. Phys. 82(1), 557 (2010) 
100. P. Fayet, Parity violation effects induced by a new gauge boson, Phys. Lett. B 96(1-2), 83 (1980)

101. C. Bouchiat and P. Fayet, Constraints on the parityviolating couplings of a new gauge boson, Phys. Lett. B 608(1-2), 87 (2005)

102. B. Holdom, Two U(1)'s and charge shifts, Phys. Lett. B 166(2), 196 (1986)

103. H. Davoudiasl, H. S. Lee, and W. J. Marciano, Dark side of Higgs diphoton decays and muon $g-2$, Phys. Rev. D 86(9), 095009 (2012)

104. H. Davoudiasl, H. S. Lee, and W. J. Marciano, "Dark" Z implications for parity violation, rare meson decays, and Higgs physics, Phys. Rev. D 85(11), 115019 (2012)

105. A. Kurylov, M. J. Ramsey-Musolf, and S. Su, Probing supersymmetry with parity-violating electron scattering, Phys. Rev. D 68, 035008 (2003)

106. G. Aad, et al. (ATLAS Collaboration), Muon reconstruction efficiency and momentum resolution of the ATLAS experiment in proton-proton collisions at $\sqrt{s}=7 \mathrm{TeV}$ in 2010, Eur. Phys. J. C 74, 3134 (2014)

107. V. Khachatryan, et al. (CMS Collaboration), Search for quark contact interactions and extra spatial dimensions using dijet angular distributions in proton-proton collisions at $\sqrt{s}=8 \mathrm{TeV}$, Phys. Lett. B 746, 79 (2015)

108. F. D. Aaron, et al. (H1 Collaboration), Search for contact interactions in $e^{ \pm} p$ collisions at HERA, Phys. Lett. B 705, $52(2011)$

109. G. Abbiendi, et al. (OPAL Collaboration), Tests of the standard model and constraints on new physics from measurements of fermion-pair production at 189-209 GeV at LEP, Eur. Phys. J. C 33, 173 (2004)

110. S. Typel and B. A. Brown, Neutron radii and the neutron equation of state in relativistic models, Phys. Rev. C 64, 027302 (2001)

111. J. Aasi, et al. (LIGO Scientific Collaboration and Virgo Collaboration), Search for gravitational waves from binary black hole inspiral, merger, and ringdown in LIGO-Virgo data from 2009-2010, Phys. Rev. D 87, 022002 (2013)

112. J. Aasi, et al. (LIGO Scientific), Enhanced sensitivity of the LIGO gravitational wave detector by using squeezed states of light, Nature Photon. 7, 613 (2013)

113. C. J. Horowitz and Kai Kadau, Breaking strain of neutron star crust and gravitational waves, Phys. Rev. Lett. 102, 191102 (2009)

114. C. J. Horowitz and J. Piekarewicz, Constraining URCA cooling of neutron stars from the neutron radius of ${ }^{208} \mathrm{~Pb}$, Phys. Rev. C 66, 055803 (2002)

115. G. Hagen, T. Papenbrock, M. Hjorth-Jensen, and D. J. Dean, Coupled-cluster computations of atomic nuclei, Rept. Prog. Phys. 77, 096302 (2014) 